\title{
Adult Hippocampal Neurogenesis along the Dorsoventral Axis Contributes Differentially to Environmental Enrichment Combined with Voluntary Exercise in Alleviating Chronic Inflammatory Pain in Mice
}

\author{
Jie Zheng, ${ }^{1}$ Ying-Ying Jiang, ${ }_{1}^{1}$ Ling-Chi Xu, ${ }^{1}$ Long-Yu Ma, ${ }^{1}$ Feng-Yu Liu, ${ }^{1}$ Shuang Cui, ${ }^{1}$ Jie Cai, ${ }^{1}$ Fei-Fei Liao, ${ }^{1}$ \\ (You Wan, ${ }^{1,2}$ and Ming Y $\mathbf{i}^{1}$ \\ ${ }^{1}$ Neuroscience Research Institute and Department of Neurobiology, School of Basic Medical Sciences, and ${ }^{2}$ Key Laboratory for Neuroscience, Ministry of \\ Education/National Health and Family Planning Commission, Peking University, Beijing 100191, P.R. China
}

Cognitive behavioral therapy, such as environmental enrichment combined with voluntary exercise (EE-VEx), is under active investigation as an adjunct to pharmaceutical treatment for chronic pain. However, the effectiveness and underlying mechanisms of EE-VEx remain unclear. In mice with intraplantar injection of complete Freund's adjuvant, our results revealed that EE-VEx alleviated perceptual, affective, and cognitive dimensions of chronic inflammatory pain. These effects of EE-VEx on chronic pain were contingent on the occurrence of adult neurogenesis in the dentate gyrus in a functionally dissociated manner along the dorsoventral axis: neurogenesis in the ventral dentate gyrus participated in alleviating perceptual and affective components of chronic pain by EE-VEx, whereas neurogenesis in the dorsal dentate gyrus was involved in EE-VEx's cognitive-enhancing effects. Chronic inflammatory pain was accompanied by decreased levels of brain-derived neurotrophic factor (BDNF) in the dentate gyrus, which were reversed by EE-VEx. Overexpression of BDNF in the dentate gyrus mimicked the effects of EE-VEx. Our results demonstrate distinct contribution of adult hippocampal neurogenesis along the dorsoventral axis to EE-VEx's beneficial effects on different dimensions of chronic pain.

Key words: adult neurogenesis; anxiety; brain-derived neurotrophic factor; chronic inflammatory pain; environmental enrichment; hippocampus

\section{Significance Statement}

Environmental enrichment combined with voluntary exercise (EE-VEx) is under active investigation as an adjunct to pharmaceutical treatment for chronic pain, but its effectiveness and underlying mechanisms remain unclear. In a mouse model of inflammatory pain, the present study demonstrates that the beneficial effects of EE-VEx on chronic pain depend on adult neurogenesis with a dorsoventral dissociation along the hippocampal axis. Adult neurogenesis in the ventral dentate gyrus participates in alleviating perceptual and affective components of chronic pain by EE-VEx, whereas that in the dorsal pole is involved in EE-VEx's cognitive-enhancing effects in chronic pain.

\section{Introduction}

Chronic pain is one of the most prevalent clinical situations and includes perceptual, affective, and cognitive dimensions (Bush-

Received Oct. 27, 2016; revised Feb. 27, 2017; accepted March 2, 2017.

Author contributions: J.Z., F.-Y.L., Y.W., and M.Y. designed research; J.Z., Y.-Y.J., L.-C.X., L.-Y.M., S.C., J.C., and F.-F.L. performed research;J.Z.,Y.-Y.J., L.-C.X., L.-Y.M., S.C., J.C., and F.-F.L. analyzed data;J.Z., Y.W., and M.Y. wrote the paper.

This work was supported by the National Basic Research Program of the Ministry of Science and Technology of China (2013CB531905, 2014CB548200, and 2015CB554503), the National Natural Science Foundation of China (81230023, 81571067, 81521063, and 21305005), the Key Project of the Chinese Ministry of Education (109003), and the "111" Project of the Ministry of Education of China (B07001). Funders have no role in experimental design, data collection, discussion, and explanation. We thank Alison Hildebrandt of the University of California, Los Angeles for help in manuscript editing.

The authors declare no competing financial interests. nell et al., 2013). Cognitive behavioral therapy, including environmental enrichment combined with voluntary exercise (EEVEx), is being investigated as an adjuvant to pharmaceutical treatments for various neurological disorders (Nithianantharajah and Hannan, 2006). Both environmental enrichment and exercise alleviate sensory hypersensitivity or other nociceptive responses in animals with neuropathic or inflammatory pain (Tall, 2009; Vachon et al., 2013), but the underlying mechanisms remain unclear.

Correspondence should be addressed to either of the following at the above address: Y. Wan, E-mail: ywan@hsc.pku.edu.cn; or M. Yi. E-mail: mingyi@bjmu.edu.cn.

https://doi.org/10.1523/JNEUROSCI.3333-16.2017

Copyright $\odot 2017$ the authors 
One striking effect of EE-VEx on the nervous system is to promote adult neurogenesis in the hippocampal dentate gyrus (DG; van Praag et al., 1999), which is at least partially mediated by upregulated local brain-derived neurotrophic factor (BDNF) (Fan et al., 2016). Adult neurogenesis modulates hippocampus-dependent functions along the dorsoventral axis: newly born neurons in the dorsal pole contribute to spatial/contextual learning whereas those in the ventral pole contribute to anxiety/depression-like behaviors (O'Leary and Cryan, 2014). The hippocampus receives both direct and indirect nociceptive inputs and in turn modulates spinal nociceptive processing through the descending pathway (Liu and Chen, 2009). Adult hippocampal neurogenesis is impaired in animals with chronic neuropathic or inflammatory pain (Duric and McCarson, 2006; Terada et al., 2008; Mutso et al., 2012; Dellarole et al., 2014; Dimitrov et al., 2014). By contrast, several antidepressants capable to promote adult hippocampal neurogenesis in depressed subjects (Eisch and Petrik, 2012) are recommended for pain management (Finnerup et al., 2015). These evidences raise the possibility that adult hippocampal neurogenesis contributes to EE-VEx's pain-relieving effects. To test this hypothesis, we observed the influence of EE-VEx on perceptual, affective, and cognitive dimensions of chronic inflammatory pain induced by intraplantar injection of complete Freund's adjuvant (CFA) in mice and examined potential roles of hippocampal neurogenesis in these processes through region-specific ablation.

\section{Materials and Methods}

Animals and housing. Male C57BL/6 mice (RRID:IMSR_JAX:000664) were provided by the Department of Laboratory Animal Sciences, Peking University Health Science Center. B6.Cg- Tg(Nes-cre) $1 \mathrm{Kln} / \mathrm{J}$ mice (RRID:IMSR_JAX:003771) were obtained from Nanjing Biomedical Research Institute of Nanjing University. All animals were housed under pathogen-free circumstances, with a $12 \mathrm{~h}$ alternating light/dark cycle and food and water available ad libitum. Mice weighing 20-30 g (5-8 weeks of age) were used at the start of experiments. All animal experiments followed the guidelines of the Committee for Research and Ethical Issues of the International Association for the Study of Pain and were approved by the Animal Care and Use Committee of Peking University Health Science Center.

The EE-VEx cage was a three-layered Plexiglas room, connected by plastic tunnels between layers, with the following sterilized toys placed in each cage: two running wheels, three tunnels of different styles, a screwtype ladder, a swing, a teeterboard, a nest made of medical cotton, and a crib. Toy arrangement remained the same in each EE-VEx cage. As the control, basic housing $(\mathrm{BH})$ cages were normal Plexiglas cages without additional toys (Fig. 1A). All animals were housed in the same room with the same bedding materials, water, and food. Mice were housed for 2 weeks before testing and remained housed in these conditions for the remainder of the study. Experimenters were blind from the groupings of mice in all experiments.

Thermal and mechanical pain thresholds. Each mouse was handled for 5 min and adapted in a Plexiglas cube for $30 \mathrm{~min}$ per day for $3 \mathrm{~d}$ before the first measurement. Thermal or mechanical pain thresholds were measured while the mouse stayed calm and awake. Paw withdrawal latencies (PWLs) to thermal stimulus were measured by a focused radiant heat ( $15 \mathrm{~W}$ of power) applied to either hindpaw of the mice (Hargreaves Method, IITC 390). PWLs were recorded three times and averaged as the thermal pain threshold. A cutoff value of $20 \mathrm{~s}$ was set to avoid possible tissue injuries.

Fifty percent paw withdrawal thresholds (50\% PWT) in response to 0.02 $1.4 \mathrm{~g}$ von Frey hairs (Stoelting) were measured using the up-and-down method (Chaplan et al., 1994). Each test began with a hair of $0.16 \mathrm{~g}$ force delivered perpendicularly to the central plantar surface of either hindpaw for 3 s. Positive responses included sudden paw withdrawal, flinching, or paw licking. The 50\% PWT of either hindpaw was calculated using the following formula: $50 \%$ PWT $(\mathrm{g})=10^{\mathrm{Xf}+\mathrm{k} \delta} / 10,000$, where Xf is the handle-marking value of the final von Frey hair used, $k$ is the tabular value for the pattern of positive/negative responses, and $\delta$ is the average interval (in log units) between von Frey hairs used.
CFA-induced inflammatory pain. Mice were anesthetized with isoflurane. The plantar surface of the left hindpaws was cleaned by $75 \%$ ethanol, before a total of $50 \mu \mathrm{l}$ of CFA was injected intraplantarly. For controls, equal volumes of normal saline were injected. Paw volumes below the elbow joint were measured with water displacement plethysmography (ZH-YLS-7B, ZS Dichuang Company).

Formalin test. Each mouse was handled for $5 \mathrm{~min}$ and adapted in a Plexiglas chamber for $30 \mathrm{~min}$ per day for $3 \mathrm{~d}$ before the test. Each mouse received an injection of $20 \mu \mathrm{l}$ of $2 \%$ formaldehyde solution into the plantar surface of left hindpaws, with its behavior videotaped in the following $60 \mathrm{~min}$. Time spent on licking and lifting the injected paw was counted, and the formalin pain score was calculated as described previously: (time lifting $+2 \times$ time licking)/(time lifting + time licking) (Zhang et al., 2014). The chamber was cleaned by $75 \%$ ethanol between tests.

Immunostaining. Mice were anesthetized with $1 \%$ pentobarbital sodium and intracardially perfused with $4 \%$ paraformaldehyde (PFA; in $0.1 \mathrm{M}$ phosphate buffer, pH 7.4). Brains were postfixed with 4\% PFA for $6 \mathrm{~h}$ and cryoprotected in 20 and $30 \%$ sucrose solutions in turn. Fifty-micrometer sections were sliced coronally using a cryostat microtome (model 1950, Leica), throughout the entire hippocampus. Free-floating sections were washed in PBS, blocked with a buffer containing $5 \%$ bull serum albumin and $0.3 \%$ Triton X-100 for $1 \mathrm{~h}$, and incubated with primary antibodies at $4^{\circ} \mathrm{C}$ for $24 \mathrm{~h}$ : mouse anti-5-bromo-2'-deoxyuridine (BrdU; 1:200, AbD, catalog \#MCA2483, Serotec; RRID:AB_808349), goat anti-doublecortin (DCX; 1:100, catalog \#sc-8066, Santa Cruz Biotechnology; RRID:AB_2088494), rabbit anti-neuronal nuclei (NeuN; 1:500, catalog \#MABN140, Millipore; RRID:AB_2571567), rabbit anti-nestin (1:200, catalog \#ab27952, Abcam; RRID:AB_776698), and rabbit anti-GFAP (1:500, catalog \#ab7260, Abcam; RRID:AB_305808). Sections were then washed in PBS and incubated with secondary antibodies at room temperature for $90 \mathrm{~min}$ : Alexa Fluor 488-conjugated goat anti-mouse IgG (1:400, catalog \#ab150113, Abcam; RRID:AB_2576208), Alexa Fluor 568-conjugated goat anti-mouse IgG (1:400, catalog \#A-11004, Thermo Fisher Scientific; RRID: AB_2534072), cy3-conjugated donkey anti-goat IgG (1:500, catalog \#305165-003, Jackson ImmunoResearch Laboratories; RRID:AB_2339464), FITC-conjugated donkey anti-goat IgG (1:500, catalog \#705-095-003, Jackson ImmunoResearch Laboratories; RRID:AB_2340400), Alexa Fluor 405conjugated goat anti-rabbit IgG (1:500, catalog \#111-475-003, Jackson ImmunoResearch Laboratories; RRID:AB_2338035), and Alexa Fluor 488conjugated goat anti-rabbit IgG [1:400, catalog \#A-11034 (also A11034), Thermo Fisher Scientific; RRID:AB_2576217]. For BrdU staining, sections were incubated in $2 \mathrm{~N} \mathrm{HCl}$ at $37^{\circ} \mathrm{C}$ for 30 min to expose epitopes in DNA and rinsed in $0.1 \mathrm{~m}$ sodium borate, $\mathrm{pH} 8.5$, before being blocked with serum. Images were taken by a laser-scanning confocal microscope (model FV1000, Olympus).

Adult neurogenesis analysis. BrdU (B9285, Sigma-Aldrich) was dissolved in $0.01 \mathrm{M}$ PBS $(10 \mathrm{mg} / \mathrm{ml})$. A total of $0.2 \mathrm{ml}$ of BrdU solution was injected intraperitoneally per day for 5 consecutive days before brain perfusion, unless otherwise specified. Immunofluorescence images were obtained by scanning a $z$-series stack at a $3 \mu \mathrm{m}$ interval throughout 50 - $\mu$ m-thick sections (model FV1000, Olympus).

For quantification of adult neurogenesis (Duric and McCarson, 2006), the number of BrdU/DCX colabeled cells in the subgranular zone (SGZ) of every third section was counted by an experimenter blinded from animal grouping. Four sections were counted for dorsal DG (dDG) or ventral DG (vDG), respectively. The cell counts were multiplied by three and added to indicate the total number of BrdU/DCX colabeled cells. Numbers of neurons in left and right DG were counted separately but averaged, since no significant differences were observed (data not shown).

For the analysis of dendrite complexity, images were zoomed in (Olympus Fluoview FV10-ASW; RRID:SCR_014215). Lines along the outer edge of the granular cell layer and $80 \mu \mathrm{m}$ away from it in the molecular layer were drawn. Intersections of DCX-labeled dendrites along the two lines were counted, respectively. The ratio of the intersection along the molecular layer and the outer edge of the granular cell layer was used to indicate the dendrite complexity of DCX-labeled immature neurons (Rosenzweig and Wojtowicz, 2011). 
Western blotting. Mouse brains were extracted, embedded in optimum cutting temperature compound (0201 08926, Leica), and frozen in liquid nitrogen immediately. Dorsal $(-1.0$ to $-2.5 \mathrm{~mm})$ or ventral $[-2.5$ to -4.0 $\mathrm{mm}$, anteroposterior (AP) to bregma] DG tissues were extracted using a puncture needle of 9 gauge in a cryostat microtome according to the stereological location (Paxinos and Franklin, 2001). After being extracted from tissues (P1201-50, Applygen Technologies), an equivalent of $80 \mu \mathrm{g}$ of cytoplasmic protein was mixed with loading buffer containing $2 \%$ SDS, $100 \mathrm{~mm}$ dithiothreitol, $10 \%$ glycerol, and $0.25 \%$ bromophenol blue. Proteins were separated in 15\% SDS-PAGE gels and transferred onto polyvinylidene fluoride membranes (ISEQ00010, Merck Millipore). The membranes were blocked with $10 \%$ defatted milk at room temperature for $2 \mathrm{~h}$, incubated with rabbit anti-BDNF antibody (1:100, catalog \#sc-546, Santa Cruz Biotechnology; RRID:AB_630940) or mouse anti- $\beta$-actin antibody (1:2000, catalog \#TA-09, ZSGB-Bio; RRID:AB_2636897) at $4^{\circ} \mathrm{C}$ for $24 \mathrm{~h}$, washed in Trisbuffered saline and Tween 20 , and incubated with horseradish peroxidaseconjugated goat anti-rabbit IgG antibody (1:2000, catalog \#111-035-003, Jackson ImmunoResearch Laboratories; RRID:AB_2313567) or horseradish peroxidase-conjugated goat anti-mouse IgG antibody (1:2000, catalog \#115035-003, Jackson ImmunoResearch Laboratories; RRID:AB_10015289) at room temperature for $1 \mathrm{~h}$. Protein bands were detected using Western blotting luminol reagent (sc-2048, Santa Cruz Biotechnology) and exposed onto $\mathrm{x}$-ray films. Bands were scanned (CanoScan LiDE 110) and quantified using Quantity One 1-D Analysis Software (version 4.6.2; RRID:SCR_014280).

ELISA. Under anesthesia with $1 \%$ pentobarbital sodium, $\sim 10 \mathrm{mg}$ tissues from the central plantar surface of left (injected) hindpaws were dissected and homogenized with RIPA lysis buffer (C1053, Applygen Technologies). Protein concentrations in the sample were determined using a BCA protein assay kit (23227, Thermo Fisher Scientific). The following inflammatory factors (Bessa et al., 2016) were measured using mouse ELISA kits: interleukin-1 $\beta$ (IL-1 $\beta$; DRE30583I), IL-6 (DRE30044I), tumor necrosis factor- $\alpha$ (TNF- $\alpha$; DRE30030I), and C-reactive protein (CRP; DRE30068I) obtained from Beijing Peak Albert Biotechnology Company. Absorbance was measured using a microplate reader (Multiskan GO, Thermo Fisher Scientific).

Open-field test. Each mouse was placed in a $60 \times 60 \times 60 \mathrm{~cm}$ box exposed to 60 lux illumination, with its activities videotaped for $5 \mathrm{~min}$. Time spent (C.Time) and distance traveled (C.Dis) in the central area $(30 \times 30 \mathrm{~cm})$ and total distance traveled (T.Dis) in the field were measured using the SMART software (version 2.5.21, Panlab, SMART Videotracking, Harvard Apparatus; RRID:SCR_002852). The box was cleaned by $75 \%$ ethanol between tests.

Elevated plus-maze test. The elevated plus-maze test was performed on the next day of the open-field test, unless otherwise noted. The maze was placed $50 \mathrm{~cm}$ above the floor in a 5 lux illuminated room and consisted of two open arms and two closed arms $(5 \times 30 \mathrm{~cm}$ and $15 \mathrm{~cm}$ wall height for the closed arms). Each mouse was placed onto the center area, heading toward the same open arm, and videotaped in the following $5 \mathrm{~min}$. Time spent (O.Time) and numbers of entries (O.Entries) into open arms and total arm entries (T.Entries) were analyzed using the SMART software. The maze was cleaned by $75 \%$ ethanol between tests.

Object-place recognition. Mice were handled for $5 \mathrm{~min}$ and adapted in a $20 \times 20 \times 20 \mathrm{~cm}$ box marked with visual cues for $30 \mathrm{~min}$ per day for $2 \mathrm{~d}$ before test. The task consisted of two phases (Whissell et al., 2013). In the sample phase, mice were placed in the box with two identical objects (two cubes, arbitrarily named as $O_{\mathrm{A}}$ and $O_{\mathrm{A}}$ ) at two different corners. Mice were allowed to explore freely for $2 \mathrm{~min}$ before they were removed from the box for another $2 \mathrm{~min}$, when the box and objects were cleaned by $75 \%$ ethanol. Pseudorandomly, one of the two objects $\left(O_{\mathrm{A}}\right)$ remained unchanged and left in the same place, with the other $\left(O_{\mathrm{B}}\right)$ moved to a new corner. For the test phase, mice were placed into the box again and allowed to explore for $2 \mathrm{~min}$. Once the mouse headed to and contacted the object by its nose, one exploration was counted. The bias score was calculated as the exploring time $\left(O_{\mathrm{B}}-O_{\mathrm{A}}\right) /\left(O_{\mathrm{B}}+O_{\mathrm{A}}\right)$.

Adeno-associated virus. The Cre-dependent viral vector was packaged by inserting the diphtheria toxin fragment A (DTA) coding sequence into a CAG-promoted pAAV-DIO-ires-hrmCherry vector (Virovek; Soumier and Sibille, 2014). When virus was injected into the DG of nestin-Cre mice, cytotoxic DTA was expressed to kill nestin-expressing neural pro- genitor cells to inhibit adult hippocampal neurogenesis. The viral vector without DTA gene insertion was used as controls.

BDNF overexpression was obtained by a pAAV-CMV-MCS-EGFP3Flag (AOV-022) vector, in which the $b d n f$ gene sequence was cloned into the reading frame between the BamHI and the HindIII sites. Packaged virus expressed BDNF-EGFP fusion protein after CMV promoters (OBIO Technology). The viral vector without $b d n f$ gene insertion was used as controls.

For stereotaxical injection, mice were anesthetized with $1 \%$ pentobarbital sodium. A total of $0.5 \mu \mathrm{l}$ of virus solution was injected into the dDG $(-1.5 \mathrm{~mm} \mathrm{AP}, \pm 1.0 \mathrm{~mm} \mathrm{ML},-1.7 \mathrm{~mm}$ DV from bregma) or vDG $(-3.7$ $\mathrm{mm} \mathrm{AP}, \pm 2.5 \mathrm{~mm} \mathrm{ML},-2.0 \mathrm{~mm}$ DV from the bregma). AAV-BDNF/ EGFP infection was confirmed after behavioral tests by EGFP expression. The effectiveness of adult neurogenesis ablation by AAV-DIO-DTAmCherry was verified by immunostaining of newborn neuron markers. Subjects with off-target expression were excluded from further analysis.

Statistical analysis. Data were presented as means \pm SEM, unless otherwise specified. All data were analyzed and plotted using IBM SPSS Statistics (version 20.0.0, IBM; RRID:SCR_002865) and GraphPad Prism (version 5.01, GraphPad Software; RRID:SCR_002798). Unpaired twotailed $t$ test, ANOVA (one-way, two-way, or repeated measures) with Bonferroni's post hoc tests, and Mann-Whitney $U$ tests were used, with $p<0.05$ as statistically significant.

\section{Results}

\section{EE-VEx attenuated thermal hyperalgesia, mechanical allodynia, and anxiety-like behaviors in mice with chronic inflammatory pain}

EE-VEx had limited effects on baseline thermal (group effect: $F_{(3,28)}=0.14, p>0.05$; time effect: $F_{(1,28)}=0.06, p>0.05$; interaction: $F_{(3,28)}=1.44, p>0.05$; Fig. $\left.1 B\right)$ and mechanical (group effect: $F_{(3,28)}=0.72, p>0.05$; time effect: $F_{(1,28)}=3.06$, $p>0.05$; interaction: $F_{(3,28)}=0.09, p>0.05$, two-way ANOVA; Fig. $1 C$ ) pain thresholds, as well as formalin-induced nociceptive behaviors (pain score: $1.16 \pm 0.04$ vs $1.21 \pm 0.03, t_{(14)}=1.36, p>$ $0.05, t$ test). By contrast, EE-VEx significantly attenuated CFAinduced thermal hyperalgesia, mainly in the chronic phase (7-17 $\mathrm{d}$ after CFA injection; group effect: $F_{(3,196)}=65.15, p<0.001$; time effect: $F_{(7,196)}=16.50, p<0.001$; interaction: $F_{(21,196)}=$ 5.92, $p<0.001 ; p<0.01$ for BH plus CFA vs EE-VEx plus CFA, ANOVA with repeated measures and Bonferroni's post hoc test; Fig. 1B). CFA-induced mechanical allodynia was also alleviated by EE-VEx (group effect: $F_{(3,196)}=55.49, p<0.001$; time effect: $F_{(7,196)}=6.47, p<0.001$; interaction: $F_{(21,196)}=1.87, p<0.05$; $p<0.05$ for BH plus CFA vs EE-VEx plus CFA, ANOVA with repeated measures and Bonferroni's post hoc test; Fig. $1 C$ ), although to a lesser extent than that for thermal hyperalgesia. Pain thresholds of the contralateral paw remained similar at all tested time points (data not shown).

Anxiety is a common comorbidity of chronic pain (Williams and Craig, 2016). We observed increased anxiety-like behaviors in mice with chronic inflammatory pain (14 d after CFA injection), indicated by less time spent (CFA effect: $F_{(1,28)}=14.29, p<$ 0.001 ; housing effect: $F_{(1,28)}=6.65, p<0.05$; interaction: $F_{(1,28)}=$ $6.59, p<0.05)$ and distance traveled (CFA effect: $F_{(1,28)}=5.13$, $p<0.05$; housing effect: $F_{(1,28)}=6.58, p<0.05$; interaction: $F_{(1,28)}=6.44, p<0.05$ ) in the central area of the open field (Fig. $1 D)$, as well as less time spent (CFA effect: $F_{(1,28)}=13.42, p<$ 0.01 ; housing effect: $F_{(1,28)}=6.38, p<0.05$; interaction: $F_{(1,28)}=$ $6.01, p<0.05)$ and fewer entries into the open arms of the elevated plus-maze (CFA effect: $F_{(1,28)}=5.86, p<0.05$; housing effect: $F_{(1,28)}=5.75, p<0.05$; interaction: $F_{(1,28)}=5.29, p<$ 0.05, two-way ANOVA with Bonferroni's post hoc test; Fig. $1 E$ ).

No statistically significant effects of EE-VEx on locomotion were observed, indicated by similar total distance (CFA effect: 

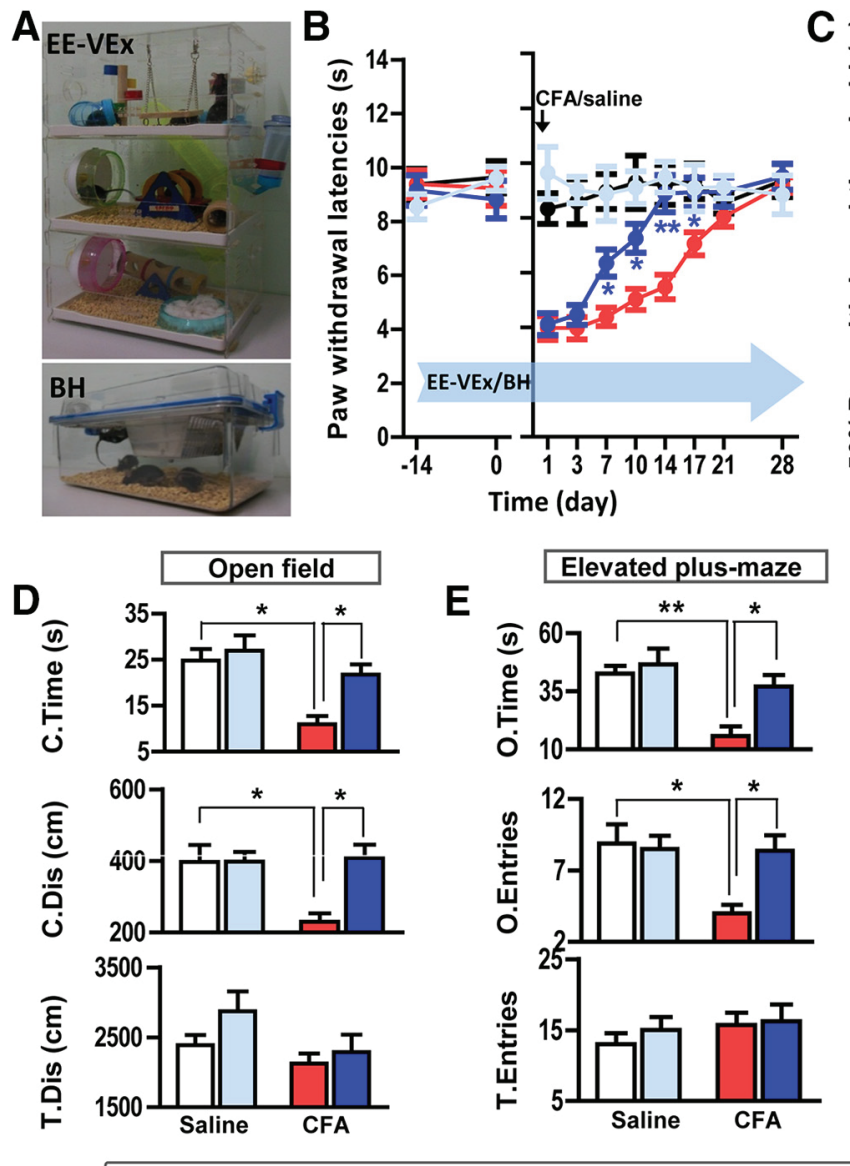
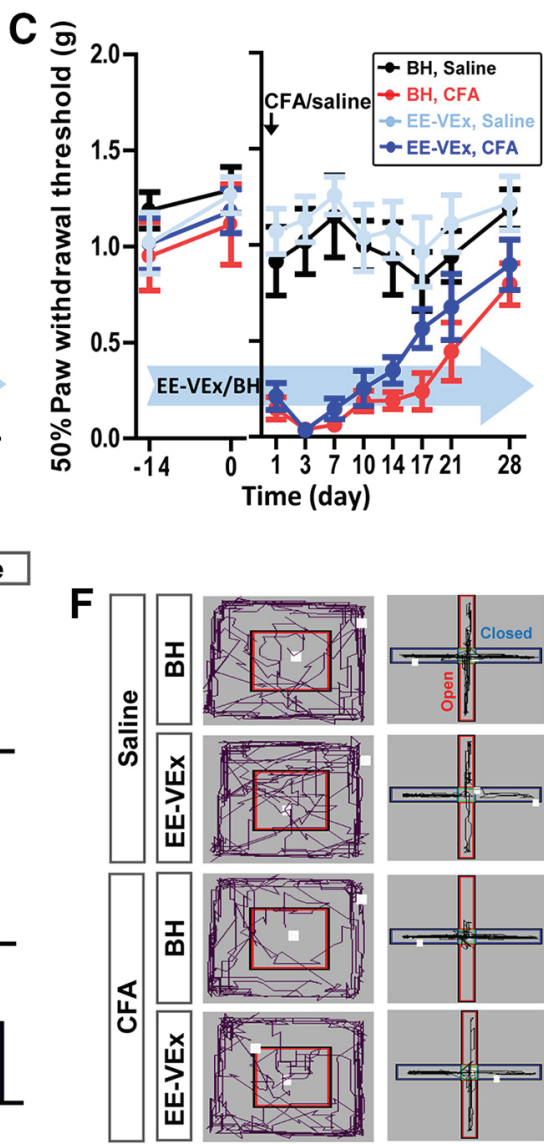

Figure 1. EE-VEx attenuated thermal hyperalgesia, mechanical allodynia, and anxiety-like behaviors in chronic inflammatory pain. $\boldsymbol{A}$, Representative photographs of EE-VEx (top) and BH (bottom) conditions. $B, C$, EE-VEx attenuated thermal hyperalgesia $(\boldsymbol{B})$ and mechanical allodynia $(\boldsymbol{C})$ in CFA-induced chronic inflammatory pain, without affecting baseline pain thresholds. $n=8$ in each group. ${ }^{*} p<0.05,{ }^{* *} p<0.001$, EE-VEx plus CFA vs BH plus CFA, ANOVA with repeated measures and Bonferroni's post hoc test. D, EE-VEx attenuated pain-induced anxiety-like behaviors in the open-field test. Persistent pain (14 d after CFA injection) decreased the time spent (top) and distance traveled (middle) in the central area of the open field, which were reversed by EE-VEx. Total distance traveled (bottom) in the field remained statistically similar across groups. $n=8$ in each group. ${ }^{*} p<0.05$, two-way ANOVA with Bonferroni's post hoc test. E, EE-VEx attenuated pain-induced anxiety-like behaviors in the elevated plus-maze test. Chronic pain decreased the time spent (top) and entries (middle) into the open arms, which were reversed by EE-VEx. Total arm entries (bottom) were not affected. $n=8$ in each group. ${ }^{*} p<0.05,{ }^{* *} p<0.01$, two-way ANOVA with Bonferroni's post hoc test. $F$, Representative exploratory tracks (black polylines) in the open field (left) and the elevated plus-maze (right).
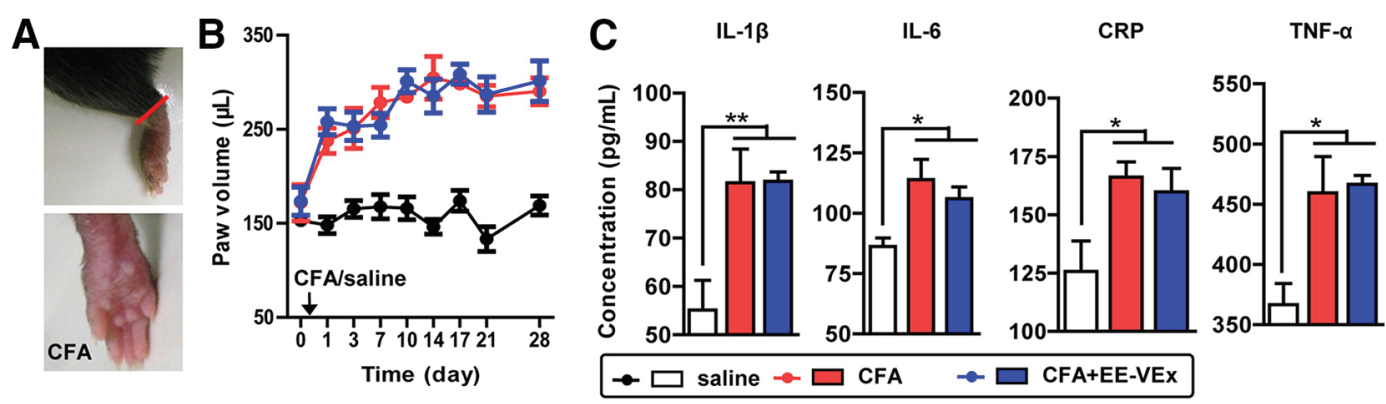

Figure 2. Limited effects of EE-VEx on CFA-induced peripheral inflammation. $A$, Representative photographs showing CFA-induced paw swelling. $\boldsymbol{B}$, CFA-induced paw swelling was not affected by EE-VEX. $n=8$ in each group. C, CFA injection upregulated local inflammatory factors, including IL- $1 \beta, \mathrm{IL}-6, \mathrm{CRP}$, and TNF- $\alpha$, in swollen paws, none of which were affected by EE-VEX. $n=8$ in each group. ${ }^{*} p<0.05,{ }^{* *} p<0.01$, one-way ANOVA.

$F_{(1,28)}=4.00, p>0.05$; housing effect: $F_{(1,28)}=2.31, p>0.05$; interaction: $F_{(1,28)}=0.57, p>0.05$; Fig. $\left.1 D\right)$ traveled in the open field and similar total arm entries (CFA effect: $F_{(1,28)}=1.24, p>$ 0.05 ; housing effect: $F_{(1,28)}=0.44, p>0.05$; interaction: $F_{(1,28)}=$ $0.16, p>0.05$, two-way ANOVA; Fig. $1 E)$ in the plus-maze.

These data indicate that EE-VEx attenuates thermal hyperalgesia, mechanical allodynia, and anxiety-like behaviors in mice with chronic inflammatory pain, without affecting their baseline nociceptive responses or exploratory behaviors.

\section{EE-VEx had limited effects on CFA-induced} peripheral inflammation

To examine whether the analgesic and anxiolytic effects of EEVEx resulted from weakened local inflammation surrounding the 


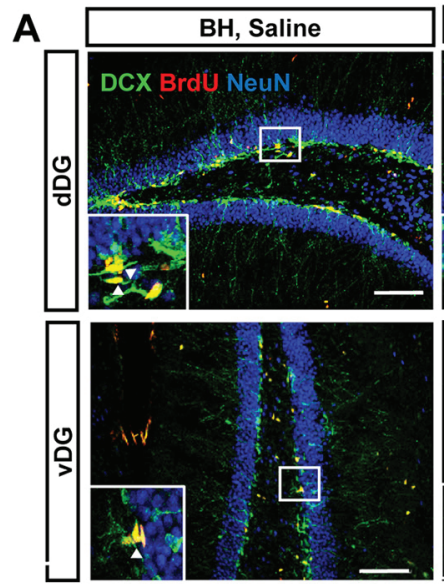

B

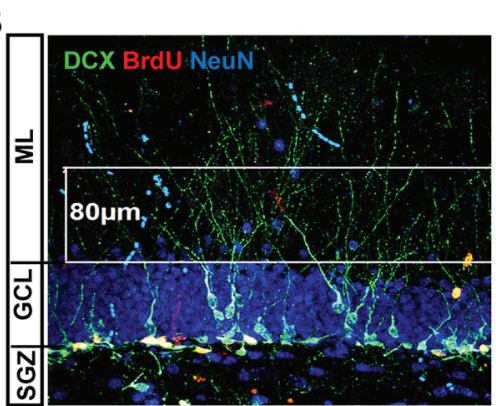

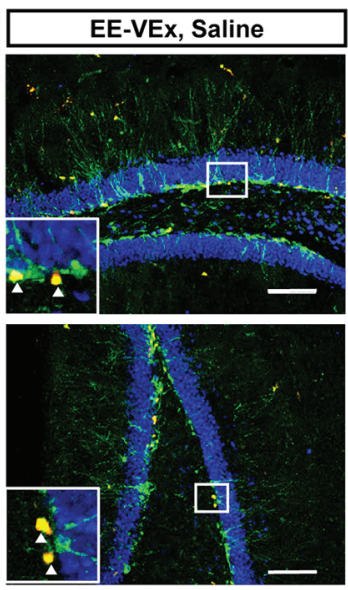

C

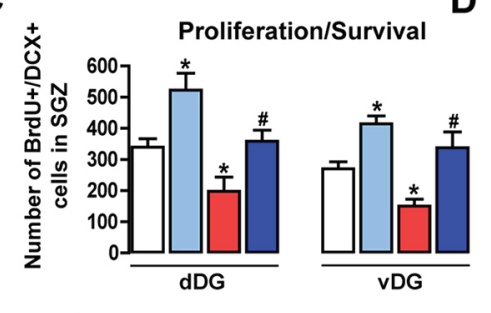

BH, CFA

$\mathrm{D}$
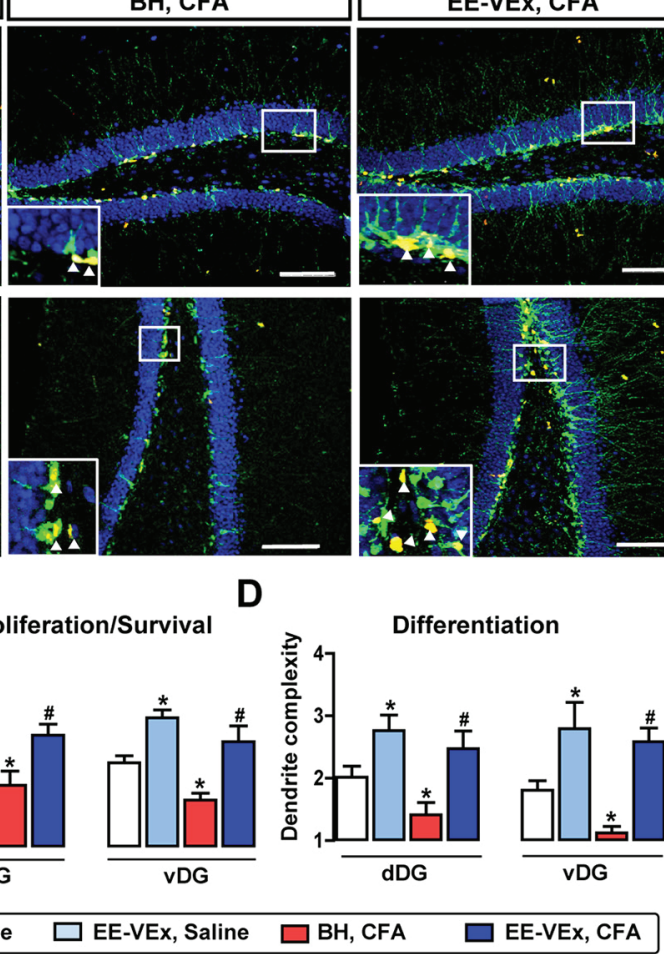

Figure 3. EE-VEx reversed impaired adult DG neurogenesis in mice with chronic inflammatory pain. $\boldsymbol{A}$, Representative immunofluorescence images showing adult neurogenesis in the dDG (top) and vDG (bottom). Magnified views of areas in the white box were shown in the bottom left corner of each image. Scale bars, $100 \mu \mathrm{m}$. Arrowheads point to BrdU/DCX colabeled neurons. $\boldsymbol{B}$, Cell population-based dendrite complexity analysis. Areas between two parallel white lines ( $80 \mu \mathrm{m}$ ) were used to analyze the dendrite complexity. $\mathrm{GCL}$, Granular cell layer; ML, molecular layer. $\boldsymbol{C}, \boldsymbol{D}$, Numbers of BrdU/DCX colabeled neurons ( $\boldsymbol{C}$ ) and dendrite complexity of DCX-labeled immature neurons (D) decreased in dDG and vDG in chronic inflammatory pain. EE-VEx promoted neurogenesis in both normal and inflammatory mice. $n=8$ in each group. ${ }^{*} p<0.05$ vs BH plus saline; ${ }^{*} p<0.05$ vs BH plus CFA, $t$ test.

injection site, we measured paw swelling (Fig. 2A) and local levels of inflammatory factors. However, CFA-induced paw swelling was not significantly affected by EE-VEx (group effect: $F_{(2,168)}=$ $311.90, p<0.001$; time effect: $F_{(8,168)}=13.92, p<0.001$; interaction: $F_{(16,168)}=3.46, p<0.001 ; p>0.05$ for CFA vs CFA plus EE-VEx, ANOVA with repeated measures and Bonferroni's post hoc test; Fig. $2 B)$. CFA elevated local levels of several inflammatory factors, including IL-1 $\beta\left(F_{(2,23)}=7.69, p<0.01\right)$, IL-6 $\left(F_{(2,23)}=5.85, p<0.01\right), \operatorname{CRP}\left(F_{(2,23)}=4.49, p<0.05\right)$, and TNF- $\alpha\left(F_{(2,23)}=7.35, p<0.01\right.$, one-way ANOVA). Again, EEVEx failed to affect these changes $(p>0.05$ for CFA vs CFA plus EE-VEx, Bonferroni's post hoc test; Fig. 2C). These results indicate limited effects of EE-VEx on local inflammation.

\section{EE-VEx reversed impaired adult hippocampal neurogenesis} in chronic inflammatory pain

Both environmental enrichment and exercise promote adult neurogenesis in the dentate gyrus (van Praag et al., 2000), which regulates spatial cognition, anxiety, and pain (Nakashiba et al., 2012; Hill et al., 2015; Apkarian et al., 2016). We asked whether chronic pain impaired hippocampal neurogenesis and, if so, whether this deficit could be reversed by EE-VEx. Considering the dorsoventral dissociation of hippocampal functions ( $\mathrm{Wu}$ and $\mathrm{Hen}, 2014$ ), we separated adult DG neurogenesis into $\mathrm{dDG}$ and $\mathrm{vDG}$ poles in all following experiments. BrdU, DCX, and NeuN were used to label newborn cells, immature granular cells, and mature neurons, respectively. The number of BrdU/DCX colabeled neurons in the SGZ of the DG was counted to quantify adult neurogenesis (Fig. $3 A$ ), as described previously (Mutso et al., 2012). The dendrite growth of DCX-labeled immature granular cells (Fig. 3B) was also analyzed (Rosenzweig and Wojtowicz, 2011).

EE-VEx promoted adult neurogenesis in normal mice, indicated by increased numbers of BrdU/DCX colabeled neurons in both dDG $\left(t_{(14)}=3.42, p<0.05\right)$ and vDG $\left(t_{(14)}=3.76, p<0.05\right.$; Fig. $3 A, C)$, as well as increased dendrite complexity of DCXlabeled immature granular cells $\left(\mathrm{dDG}: t_{(14)}=2.43, p<0.05\right.$; vDG: $t_{(14)}=2.18, p<0.05$, EE-VEx plus saline vs $\mathrm{BH}$ plus saline, $t$ test; Fig. 3D). Chronic (10-14 d after CFA injection) but not acute (3-7 d; data not shown) inflammatory pain decreased both the number of BrdU/DCX colabeled neurons (dDG: $t_{(14)}=4.38$, $p<0.01$; vDG: $t_{(14)}=3.96, p<0.01$; Fig. $\left.3 A, C\right)$ and the dendrite complexity of DCX-labeled immature granular cells (dDG: $t_{(14)}=2.24, p<0.05$; vDG: $t_{(14)}=3.81, p<0.05, t$ test, BH plus CFA vs $\mathrm{BH}$ plus saline; Fig. $3 D)$. These deficits were reversed by EE-VEx (BrdU/DCX colabeled neurons: dDG: $t_{(14)}=3.36, p<$ 0.01; vDG: $t_{(14)}=3.73, p<0.05$; Fig. $3 C$; dendrite complexity: dDG: $t_{(14)}=3.18, p<0.05$; vDG: $t_{(14)}=6.45, p<0.01$, $t$ test; Fig. $3 D)$. Thus, EE-VEx reverses impaired adult hippocampal neurogenesis in chronic inflammatory pain.

\section{Selective ablation of adult DG neurogenesis}

To directly test whether adult DG neurogenesis contributed to EE-VEx's beneficial effects on chronic pain, we adopted a Credependent AAV to selectively ablate newborn neurons with DTA, a neurotoxin, in nestin-Cre mice (Soumier and Sibille, 2014; Fig. $4 A$ ). Virus infection was verified by restricted expression of mCherry within the SGZ of dDG or vDG $21 \mathrm{~d}$ after AAVmCherry injection (Fig. $4 B$ ). A total of $39.6 \pm 8.2 \%$ of mCherry- 

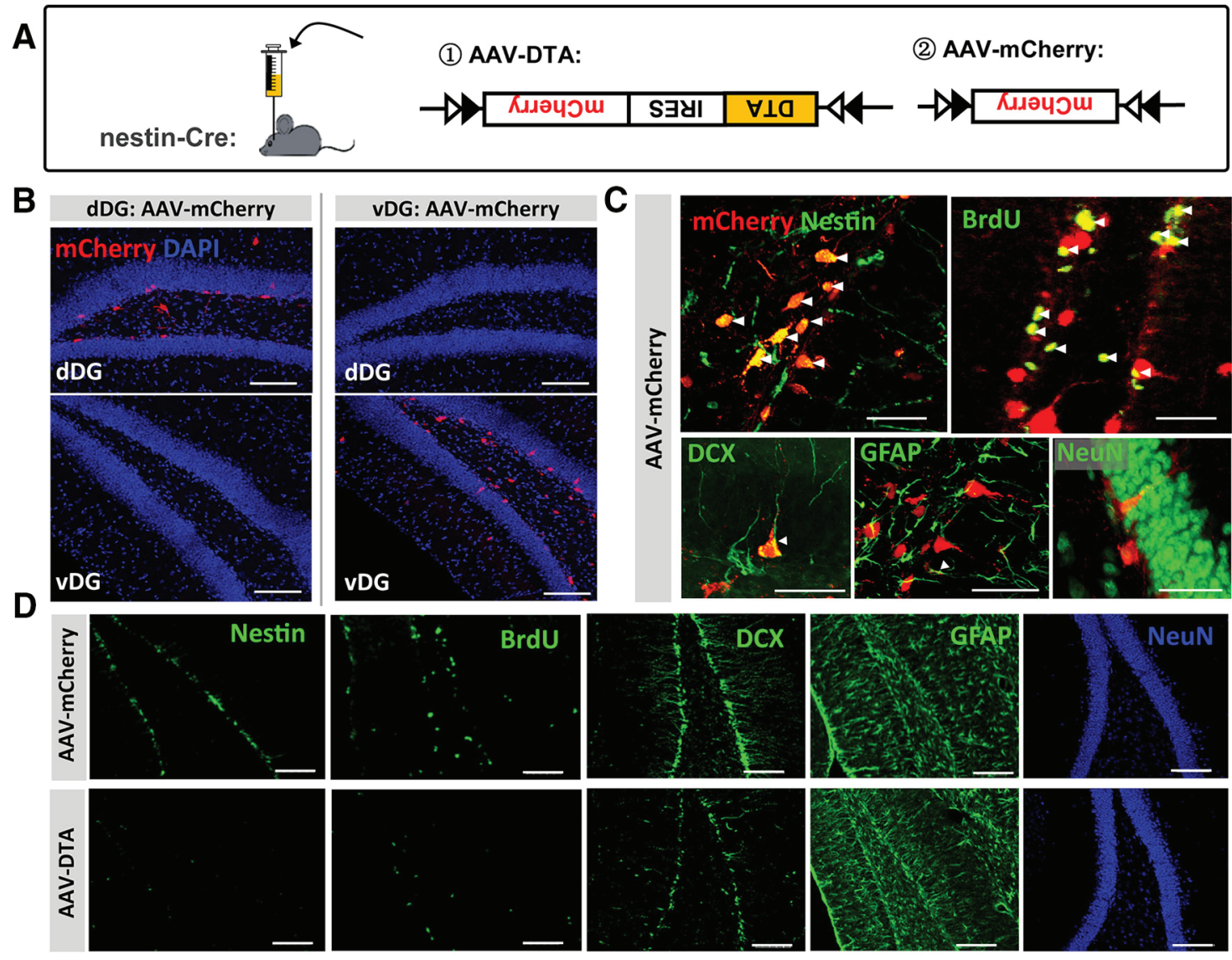

E

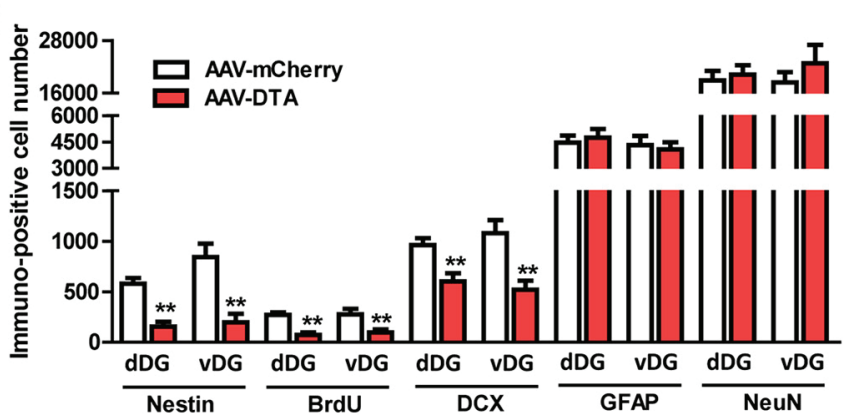

$\mathbf{F}$
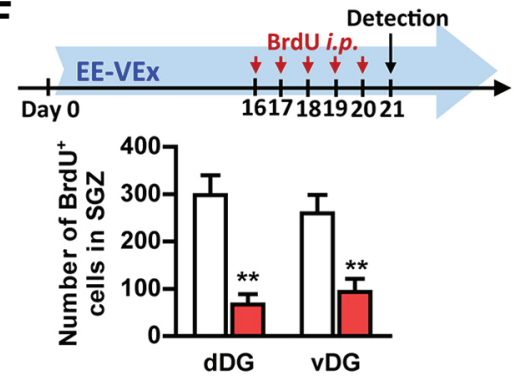

Figure 4. Selective ablation of adult DG neurogenesis by Cre-dependent viral vectors. $A$, Cre-dependent AAV-DTA (left) was applied to inhibit adult neurogenesis in nestin-Cre mice. AAV-mCherry (right) was used as the control virus. $\boldsymbol{B}$, Restricted $\mathrm{mCherry}$ expression in the SGZ of dDG (left) or vDG (right). Subjects with off-target expression were excluded from additional analysis. Scale bars, $100 \mu \mathrm{m}$. C, Representative double-labeling images of mCherry (expressed by AAV-mCherry) with nestin, BrdU, DCX, GFAP, or NeuN. $n=5$ in each group. Scale bars, $50 \mu \mathrm{m}$. Arrowheads point to colabeled neurons. $\boldsymbol{D}, \boldsymbol{E}$, AAV-DTA decreased nestin-, BrdU-, and DCX-positive cells in the dDG or vDG, without affecting GFAP- or NeuN-positive cells. $n=10$ in each group. Scale bars, $100 \mu \mathrm{m}$. $F$, AAV-DTA decreased BrdU-positive newborn cells in the SGZ of dDG or vDG under EE-VEX, with BrdU injected intraperitoneally $16-20 \mathrm{~d}$ after virus injection. $n=5$ in each group. ${ }^{* *} p<0.01, t$ test.

positive cells colabeled with nestin (marker of neural progenitor cells), $79.6 \pm 17.4 \%$ with BrdU, and $15.0 \pm 8.5 \%$ with DCX, but only $3.8 \pm 3.2 \%$ colabeled with GFAP (marker of astrocytes and neural stem cells). Few mCherry-positive cells were colabeled with NeuN (marker of mature neurons). Specific virus infection in newborn neurons was verified by high levels of mCherry/BrdU but nearly absent mCherry/NeuN colabeling (Fig. 4C).

In mice under $\mathrm{BH}, 3$ weeks after virus injection, AAV-DTA decreased $>60 \%$ nestin-positive cells $\left(\mathrm{dDG}: t_{(18)}=6.46, p<0.01\right.$; vDG: $\left.t_{(18)}=7.32, p<0.01\right),>75 \%$ BrdU-positive cells (dDG: $t_{(18)}=7.42, p<0.01$; vDG: $\left.t_{(18)}=3.14, p<0.01\right)$, and $>25 \%$ DCX-positive cells (dDG: $t_{(18)}=4.31, p<0.01$; vDG: $t_{(18)}=3.38$, $p<0.01)$ in the DG. No obvious changes were observed in GFAPpositive cells (dDG: $t_{(18)}=0.35, p>0.05$; vDG: $t_{(18)}=0.26, p>$
$0.05)$ or NeuN-positive mature granular cells (dDG: $t_{(18)}=0.63, p>$ 0.05 ; vDG: $t_{(18)}=0.75, p>0.05$, $t$ test; Fig. $\left.4 D, E\right)$.

The effectiveness of adult neurogenesis ablation by AAV-DTA under EE-VEx was verified by significantly decreased presence of BrdU-positive cells in the SGZ of dDG $\left(t_{(8)}=4.11, p<0.01\right)$ or $\operatorname{vDG}\left(t_{(8)}=4.08, p<0.01, t\right.$ test; Fig. $\left.4 F\right)$.

Adult neurogenesis in the vDG but not in the dDG contributed to the analgesic effect of EE-VEx in chronic inflammatory pain

We next examined how neurogenesis ablation modulated EEVEx and pain behaviors (Fig. 5A). Ablating adult neurogenesis in the dDG did not affect baseline thermal pain thresholds (group effect: $F_{(3,16)}=0.43, p>0.05$; time effect: $F_{(1,16)}=$ 

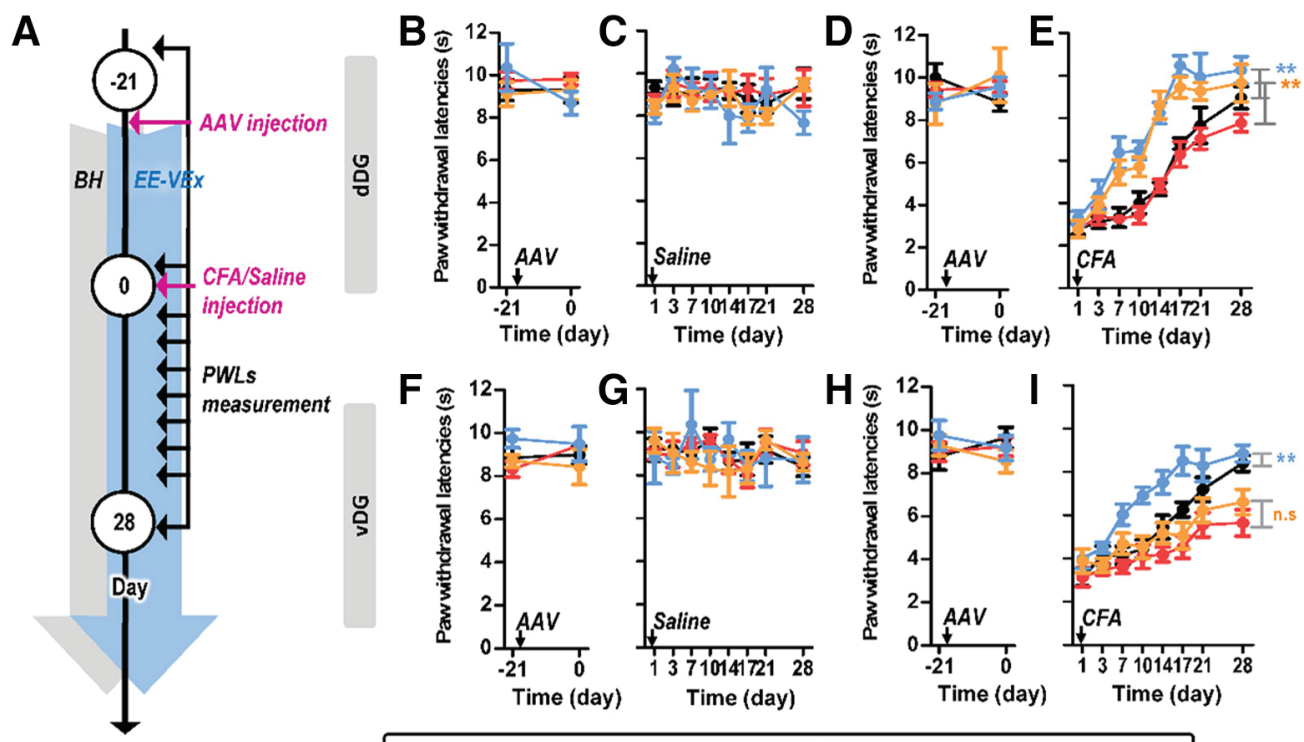

BH, AAV-mCherry $\rightarrow$ BH, AAV-DTA $\approx$ EE-VEx, AAV-mCherry $\approx$ EE-VEX, AAV-DTA

Figure 5. Adult neurogenesis ablation in the VDG but not in the dDG blocked the analgesic effect of EE-VEx in chronic inflammatory pain. $A$, Diagram showing time line of experiments. Mice were housed in EE-VEx or BH after virus injection and then injected intraplantarly with saline or CFA $21 \mathrm{~d}$ after virus injection into dDG or vDG. Mice PWLs to thermal stimulus were measured on days 1,3 , $7,10,14,17,21$, and 28 after intraplantar CFA or saline injection. $\boldsymbol{B}-\boldsymbol{E}$, Adult dDG neurogenesis ablation had limited effects on thermal pain thresholds at baseline $(n=8$ in each group; $\boldsymbol{B}, \boldsymbol{D})$, after normal saline injection ( $n=5$ in each group; $\boldsymbol{C}$, or after CFA injection ( $n=8$ in each group; $\boldsymbol{E}$ ) under either BH or EE-VEx. $\boldsymbol{F}-\boldsymbol{I}$, Ablating vDG neurogenesis did not affect thermal pain thresholds at baseline $(n=8$ in each group; $\boldsymbol{F}, \boldsymbol{H})$ or after normal saline injection $(n=5$ in each group; $\boldsymbol{G})$ but aggravated CFA-induced thermal hyperalgesia and blocked the analgesic effect of EE-VEx $(n=8$ in each group; $I) .{ }^{* *} p<0.01$, ANOVA with repeated measures and Bonferroni's post hoc test. n.s, Statistically nonsignificant.

0.59, $p>0.05$; interaction: $F_{(3,16)}=0.94, p>0.05$, Fig. $5 B$; group effect: $F_{(3,28)}=0.33, p>0.05$; time effect: $F_{(1,28)}=0.17$, $p>0.05$; interaction: $F_{(3,28)}=1.74, p>0.05$, two-way ANOVA; Fig. 5D), PWLs after saline injection (group effect: $F_{(3,112)}=1.73, p>0.05$; time effect: $F_{(7,112)}=1.15, p>0.05$; interaction: $F_{(21,112)}=0.69, p>0.05$, ANOVA with repeated measures; Fig. $5 C$ ), or thermal hyperalgesia after CFA injection under either BH or EE-VEx (group effect: $F_{(3,196)}=88.06$, $p<0.001$; time effect: $F_{(7,196)}=183.50, p<0.001$; interaction: $F_{(21,196)}=5.32, p<0.001 ; p<0.01$ for BH plus AAV-mCherry vs EE-VEx plus AAV-mCherry and for BH plus AAV-DTA vs EE-VEx plus AAV-DTA, ANOVA with repeated measures and Bonferroni's post hoc test; Fig. $5 E$ ). Thus, the analgesic effect of EE-VEx in chronic pain is not significantly affected by neurogenesis ablation in the dDG.

Similar to dDG, ablating vDG neurogenesis did not affect baseline thermal pain thresholds (group effect: $F_{(3,16)}=2.60, p>$ 0.05 ; time effect: $F_{(1,16)}=0.17, p>0.05$; interaction: $F_{(3,16)}=$ $0.57, p>0.05$; Fig. 5F; group effect: $F_{(3,28)}=0.47, p>0.05$; time effect: $F_{(1,28)}=0.02, p>0.05$; interaction: $F_{(3,28)}=0.75, p>$ 0.05, two-way ANOVA; Fig. $5 H)$ or PWLs after saline injection (group effect: $F_{(3,112)}=0.19, p>0.05$; time effect: $F_{(7,112)}=0.98$, $p>0.05$; interaction: $F_{(21,112)}=0.56, p>0.05$, ANOVA with repeated measures; Fig. $5 G$ ). By sharp contrast, neurogenesis ablation in the vDG abolished the beneficial effect of EE-VEx on the recovery from the CFA-induced thermal hyperalgesia (group effect: $F_{(3,196)}=39.32, p<0.001$; time effect: $F_{(7,196)}=34.94, p<$ 0.001 ; interaction: $F_{(21,196)}=1.77, p<0.05 ; p<0.01$ for $\mathrm{BH}$ plus AAV-mCherry vs EE-VEx plus AAV- mCherry, but $p>0.05$ for $\mathrm{BH}$ plus AAV-DTA vs EE-VEx plus AAV-DTA, ANOVA with repeated measures and Bonferroni's post hoc test; Fig. 5I). Note that neurogenesis ablation in the vDG even aggravated CFAinduced thermal hyperalgesia under $\mathrm{BH}(p<0.05$ for $\mathrm{BH}$ plus AAV-mCherry vs BH plus AAV-DTA; Fig. 5I).

These results indicate that adult neurogenesis in the vDG but not in the dDG contributes to EE-VEx's analgesic effects in chronic inflammatory pain.

\section{Adult neurogenesis in the vDG but not in the dDG contributed to the anxiolytic effect of EE-VEx in chronic inflammatory pain}

We also evaluated the effect of neurogenesis ablation on paininduced anxiety. Two weeks after CFA injection, adult dDG neurogenesis ablation did not affect the anxiolytic effects of EE-VEx in chronic pain (C.Time: virus effect: $F_{(1,16)}=0.02, p>0.05$; housing effect: $F_{(1,16)}=15.65, p<0.01$; interaction: $F_{(1,16)}=$ $0.43, p>0.05$; C.Dis: virus effect: $F_{(1,16)}=0.01, p>0.05$; housing effect: $F_{(1,16)}=14.77, p<0.01$; interaction: $F_{(1,16)}=0.02, p>$ 0.05 ; O.Time: virus effect: $F_{(1,16)}=0.01, p>0.05$; housing effect: $F_{(1,16)}=22.12, p<0.001$; interaction: $F_{(1,16)}=0.85, p>0.05$; O.Entries: virus effect: $F_{(1,16)}=0.68, p>0.05$; housing effect: $F_{(1,16)}=34.06, p<0.001$; interaction: $F_{(1,16)}=2.92, p>0.05$, two-way ANOVA with Bonferroni's post hoc test; Fig. $6 A, B$ ).

By contrast, ablating adult neurogenesis in the vDG significantly attenuated the anxiolytic effects of EE-VEx in chronic pain (14 d after CFA injection) compared with those without ablation (C.Time: virus effect: $F_{(1,14)}=5.72, p<0.05$; housing effect: $F_{(1,14)}=11.08, p<0.01$; interaction: $F_{(1,14)}=4.60, p<$ 0.05; C.Dis: virus effect: $F_{(1,14)}=4.06, p<0.05$; housing effect: $F_{(1,14)}=19.35, p<0.001$; interaction: $F_{(1,14)}=6.89$, $p<0.05$; O.Time: virus effect: $F_{(1,14)}=5.06, p<0.05$; housing effect: $F_{(1,14)}=21.92, p<0.001$; interaction: $F_{(1,14)}=6.16$, $p<0.05$; O.Entries: virus effect: $F_{(1,14)}=7.83, p<0.05$; housing effect: $F_{(1,14)}=58.93, p<0.001$; interaction: $F_{(1,14)}=$ 9.43, $p<0.05$, two-way ANOVA with Bonferroni's post hoc test; Fig. $6 C, D)$. EE-VEx no longer showed significant anxiolytic effects in mice with adult vDG neurogenesis ablation ( $p>0.05$ for C.Time, C.Dis, O.Time, and O.Entries; EE-VEx plus AAV-DTA vs BH plus AAV-DTA, Bonferroni's post hoc test; Fig. 6C,D). 

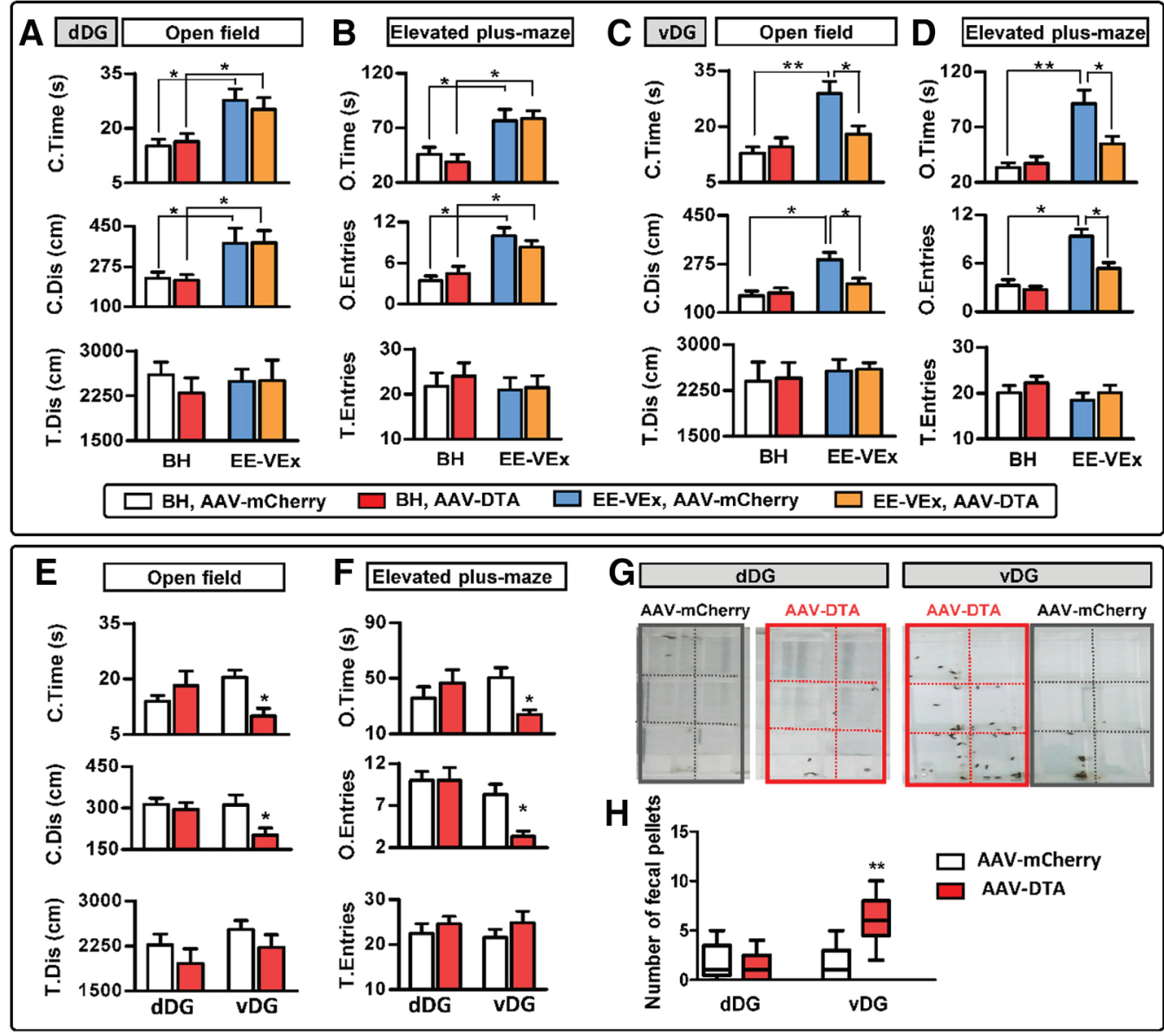

Figure 6. Adult neurogenesis ablation in the vDG blocked the anxiolytic effect of EE-VEx in chronic inflammatory pain. $A, B$, Neurogenesis ablation in the dDG had limited effects on anxiety-like behaviors in open-field $(\boldsymbol{A})$ and elevated plus-maze $(\boldsymbol{B})$ tests $14 \mathrm{~d}$ after CFA injection. $\boldsymbol{C}, \boldsymbol{D}$, Adult neurogenesis ablation in the vDG abolished the anxiolytic effect of EE-VEx in chronic pain. $n=8$ in each group. ${ }^{*} p<0.05,{ }^{* *} p<0.01$, two-way ANOVA with Bonferroni's post hoc test. $\boldsymbol{E}, \boldsymbol{F}$, Adult neurogenesis ablation in the vDG but not in the dDG aggravated anxiety-like behaviors in mice under BH (28 d after (FA injection). $n=8$ in each group. T.Dis and T.Entries remained similar in all groups. ${ }^{*} p<0.05, t$ test. $\boldsymbol{G}, \boldsymbol{H}$, Adult neurogenesis ablation in the vDG but not in the dDG increased defecation during habituation and testing phases of PWL measurement. Data were expressed as minimum-median-maximum. $n=9$ in each group. ${ }^{* *} p<0.01$, Mann-Whitney $U$ test.

In all tests, T.Dis in the open field (Fig. $6 A, C$ ) and T.Entries in the elevated plus-maze (Fig. $6 B, D$ ) remained statistically similar (two-way ANOVA), indicating similar exploratory behaviors.

We failed to observe anxiogenic effects of neurogenesis ablation on anxiety-like behaviors $14 \mathrm{~d}$ after CFA injection under BH ( $p>0.05$ for C.Time, C.Dis, O.Time, and O.Entries; BH plus AAV-DTA vs BH plus AAV-mCherry, Bonferroni's post hoc test; Fig. $6 A-D$ ). This could result from floor effects, given the high level of anxiety-like behaviors at this time point (Fig. $1 D, E)$. We examined mouse behaviors $28 \mathrm{~d}$ after CFA injection, when thermal hyperalgesia was still present in mice with vDG neurogenesis ablation (Fig. $5 F$ ). Again, vDG (C.Time: $t_{(16)}=2.87, p<0.05$; C.Dis: $t_{(16)}=2.22, p<0.05$; O.Time: $t_{(16)}=2.14, p<0.05$; O.Entries: $\left.t_{(16)}=2.17, p<0.05\right)$ but not dDG (C.Time: $t_{(16)}=$ $0.10, p>0.05$; C.Dis: $t_{(16)}=0.10, p>0.05$; O.Time: $t_{(16)}=0.86$, $p>0.05$; O.Entries: $t_{(16)}=0.01, p>0.05, t$ test) neurogenesis ablation aggravated anxiety-like behaviors (Fig. $6 E, F$ ).

Interestingly, we noticed significantly increased defecation of mice with adult vDG but not dDG neurogenesis ablation during habituation and test sessions of PWL measurements (dDG: $U=33.00, p>0.05$; vDG: $U=4.50, p<0.01$, MannWhitney $U$ test; Fig. $6 G, H)$, also indicating aggravated anxiety-like behaviors.

Together, these results parallel the aforementioned findings on thermal hyperalgesia and indicate that adult neurogenesis in the vDG but not in the dDG contributes to EE-VEx's anxiolytic effects in chronic inflammatory pain.

\section{Overxpressing BDNF in the vDG produced analgesic and}

anxiolytic effects in chronic inflammatory pain

EE-VEx upregulates hippocampal BDNF (Vedovelli et al., 2011), which promotes the survival and maturation of adult newborn neurons and improves learning and memory (Fan et al., 2016). We next asked whether impaired neurogenesis in chronic pain was accompanied by decreased local BDNF levels in the DG. $\mathrm{BDNF}$ levels were downregulated in both $\mathrm{dDG}\left(t_{(12)}=3.20, p<\right.$ $0.01)$ and vDG $\left(t_{(12)}=3.13, p<0.01, t\right.$ test $)$ in BH groups $14 \mathrm{~d}$ after CFA injection (Fig. 7A). EE-VEx upregulated BDNF in the DG of both normal (dDG: $t_{(12)}=2.42, p<0.05$; vDG: $t_{(12)}=$ $2.27, p<0.05$ ) and inflammatory (dDG: $t_{(12)}=2.45, p<0.05$; vDG: $t_{(12)}=3.95, p<0.01, t$ test; Fig. $\left.7 A\right)$ mice.

To further confirm the dorsoventral dissociation of DG in pain and EE-VEx, we overexpressed BDNF with an AAV vector in the dDG or the vDG. Significantly elevated BDNF levels were detected 2 weeks after virus injection (dDG: $t_{(8)}=2.82, p<0.05$; vDG: $t_{(8)}=3.40, p<0.01, t$ test; Fig. $\left.7 B, C\right)$. BDNF overexpression promoted local neurogenesis in chronic inflammatory pain, indicated by increased numbers of BrdU-positive cells in the SGZ $\left(\mathrm{dDG}: t_{(12)}=3.15, p<0.05\right.$; vDG: $t_{(12)}=2.76, p<0.05$; Fig. $7 D, E)$ as well as increased dendrite complexity of DCX-labeled 

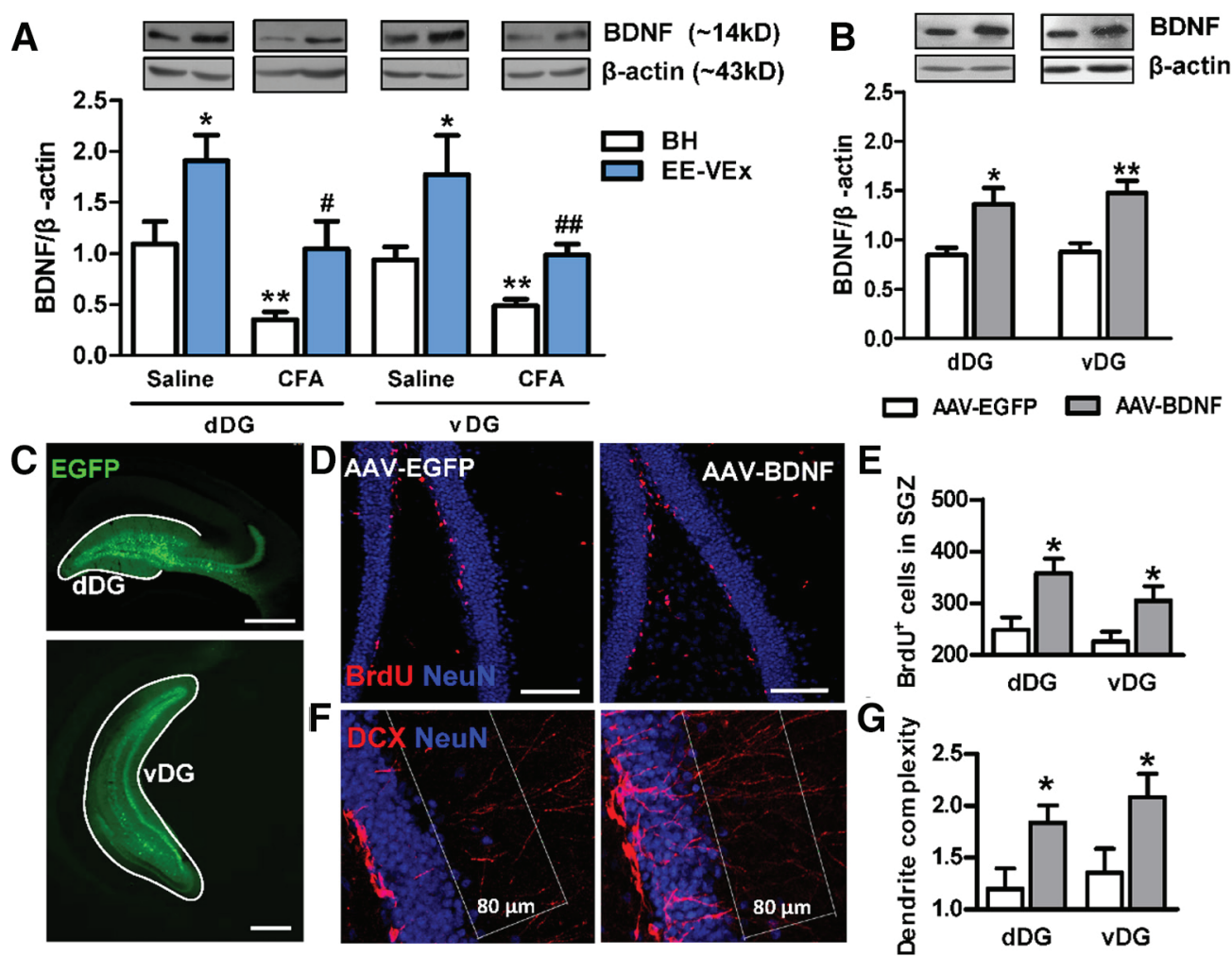

Figure 7. BDNF overexpression promoted adult DG neurogenesis. $A$, Decreases of BDNF levels in $\mathrm{DDG}$ or vDG of mice with chronic inflammatory pain were reversed by EE-VEx. Representative Western blots of $B D N F$ were shown above the corresponding histogram. $n=7$ in each group. ${ }^{*} p<0.05$, ${ }^{* *} p<0.01$ vs $B$ H plus saline; ${ }^{\#} p<0.05$, ${ }^{\# \#} p<0.01$ vs $B H$ plus $(F A, t$ test. $B, 0$ verexpressing BDNF with viral vectors. $n=5$ in each group. ${ }^{*} p<0.05,{ }^{* *} p<0.01, t$ test. C, Representative images showing virus expression (indicated by EGFP) in the dDG (top) or the vDG (bottom). Scale bars, $500 \mu \mathrm{m} . \boldsymbol{D}, \boldsymbol{E}$, BDNF overexpression increased the number of BrdU-positive cells in mice with chronic inflammatory pain. $F, G, B D N F$ overexpression increased the dendrite complexity of DCX-labeled immature granular cells in mice with chronic inflammatory pain. Scale bars, $100 \mu \mathrm{m} . n=7$ in each group. ${ }^{*} p<0.05, t$ test.

immature neurons (dDG: $t_{(12)}=2.43, p<0.05$; vDG: $t_{(12)}=$ 2.24, $p<0.05$, $t$ test; Fig. $7 F, G)$.

Consistent with the findings above, BDNF overexpression in the dDG affected neither baseline thermal pain thresholds $\left(t_{(16)}=0.92, p>0.05\right.$; Fig. $\left.8 A\right)$ nor thermal hyperalgesia developed after CFA injection (virus effect: $F_{(1,112)}=0.37, p>0.05$; time effect: $F_{(7,112)}=93.77, p<0.001$; interaction: $F_{(7,112)}=0.34$, $p>0.05$, ANOVA with repeated measures; Fig. $8 B$ ). By contrast, BDNF overexpression in the vDG attenuated thermal hyperalgesia in chronic pain (virus effect: $F_{(1,126)}=44.89, p<0.001$; time effect: $F_{(7,126)}=50.61, p<0.001$; interaction: $F_{(7,126)}=3.11, p<$ 0.01 , ANOVA with repeated measures and Bonferroni's post hoc test; Fig. $8 D$ ), without affecting baseline thermal pain thresholds $\left(t_{(18)}=0.63, p>0.05, t\right.$ test; Fig. $\left.8 C\right)$. PWLs contralateral to the CFA-injected paw remained similar across groups (data not shown).

Furthermore, $14 \mathrm{~d}$ after CFA injection, BDNF overexpression in the vDG (C.Time: $t_{(18)}=3.56, p<0.01$; C.Dis: $t_{(18)}=2.65, p<$ 0.05; O.Time: $t_{(18)}=2.52, p<0.05$; O.Entries: $t_{(18)}=2.69, p<$ 0.05 ) but not in the $\mathrm{dDG}$ (all $p$ values $>0.05, t$ test) alleviated anxiety-like behaviors in chronic pain (Fig. $8 E, F$ ).

These data indicate BDNF as a possible molecular substrate underlying EE-Vex and further confirm the differential effect of dDG and vDG in EE-VEx's analgesic and anxiolytic effects.

Adult neurogenesis in the dDG contributed to cognitiveenhancing effects of EE-VEx in chronic inflammatory pain We finally examined the possible role of dDG neurogenesis in inflammatory pain and/or EE-VEx. Previous studies have shown that the dDG participates in spatial and contextual cognition (Sahay and Hen, 2007). Cognitive impairments frequently accompany chronic pain (Apkarian et al., 2011; Mutso et al., 2012), prompting us to hypothesize that EE-VEx enhances cognitive abilities in chronic pain through dDG neurogenesis.

The object-place recognition task (Fig. 9A) requires adult neurogenesis in the dorsal hippocampus (Whissell et al., 2013). We observed impaired task performance in mice with chronic inflammatory pain, indicated by decreased bias scores toward objects removed to novel places in the test phase $\left(F_{(2,23)}=5.51\right.$, $p<0.01 ; p<0.05$ for saline vs CFA, one-way ANOVA with Bonferroni's post hoc test; Fig. 9B). EE-VEx reversed this impairment ( $p<0.05$ for CFA vs CFA plus EE-VEx; Fig. $9 B$ ), which was blocked by adult neurogenesis ablation in the $\mathrm{dDG}\left(t_{(14)}=2.75\right.$, $p<0.05)$ but not in the vDG $\left(t_{(14)}=0.91, p>0.05\right.$, $t$ test; Fig. $9 C)$. Consistently, BDNF overexpression in the dDG $\left(t_{(14)}=\right.$ $2.21, p<0.05)$ but not in the $\mathrm{vDG}\left(t_{(14)}=1.31, p>0.05, t\right.$ test $)$ attenuated the object-place cognitive impairment in chronic inflammatory pain (Fig. 9D). These results indicate that adult neurogenesis in the dDG but not in the VDG contributes to EE-VEx's cognitive-improving effects in chronic inflammatory pain.

\section{Discussion}

Mechanisms of EE-VEx's pain-relieving effects

Pain encompasses perceptual, affective, and cognitive dimensions (Williams and Craig, 2016). Previous work has shown alleviated long-term pain hypersensitivity or allodynia in chronic neuropathic or inflammatory pain with EE-VEx (Gabriel et al., 2010; Vachon et al., 2013), which is confirmed in the present 


\section{dDG}

A

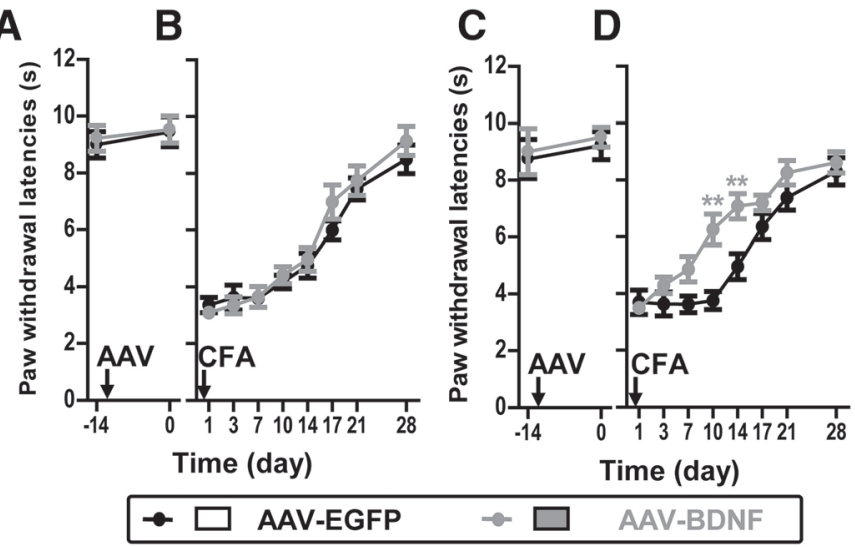

VDG

$\mathbf{E}$
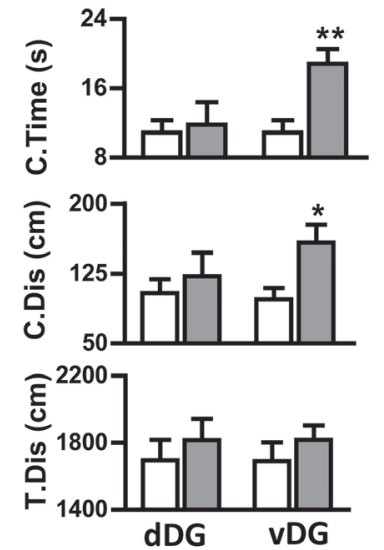

Elevated plus-maze

$\mathbf{F}$
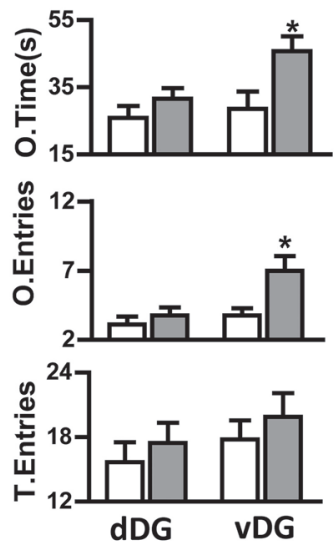

Figure 8. BDNF overexpression in the vDG but not in the dDG alleviated thermal hyperalgesia and reversed anxiety-like behaviors in chronic pain. $\boldsymbol{A}, \boldsymbol{B}, \mathrm{BDNF}$ overexpression in the dDG did not affect baseline thermal pain thresholds $(\boldsymbol{A})$ or $(F A$-induced thermal hyperalgesia $(\boldsymbol{B}) . n=9$ in each group. $\boldsymbol{C}, \boldsymbol{D}, \mathrm{BDNF}$ overexpression in the vDG did not affect baseline thermal pain thresholds $(\boldsymbol{C})$ but attenuated CFA-induced chronic thermal hyperalgesia $(\boldsymbol{D}) . n=10$ in each group. ${ }^{* *} p<0.01$, ANOVA with repeated measures and Bonferroni's post hoc test. $\boldsymbol{E}$, $\boldsymbol{F}$, AAV-BDNF expression in the vDG but not in the dDG reversed anxiety-like behaviors of mice with chronic pain, indicated by increased C.Time and C.Dis in the open field $(\boldsymbol{E})$ and increased 0 .Time and 0 .Entries in the elevated plus-maze $(\boldsymbol{F}) . n=9$ in dDG groups, $n=10$ in vDG groups. ${ }^{*} p<0.05,{ }^{* *} p<0.01, t$ test.
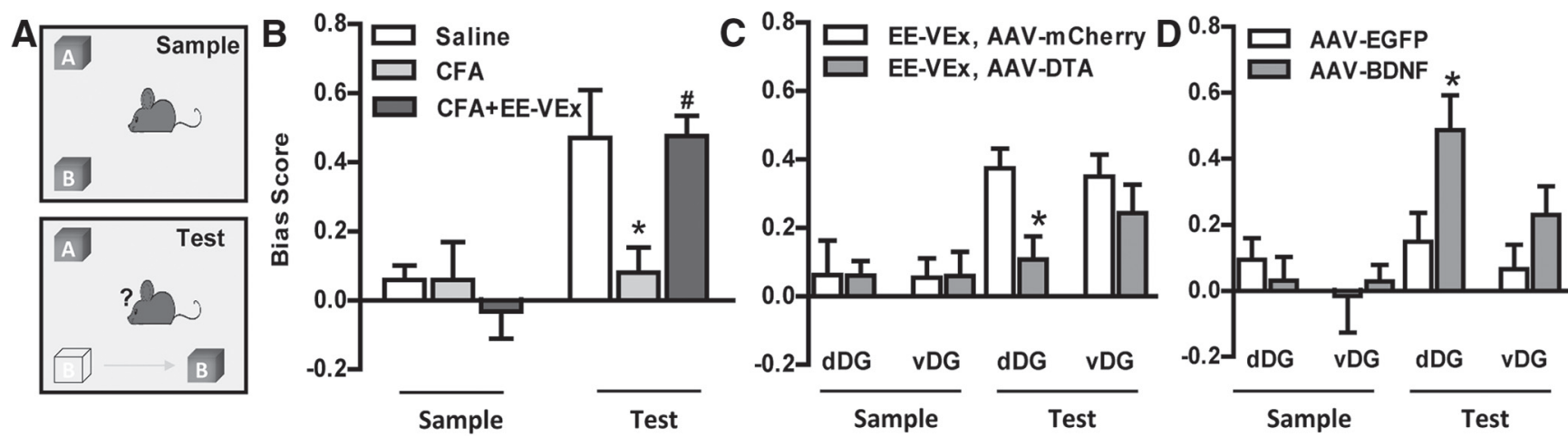

Figure 9. Adult neurogenesis in the $\mathrm{dDG}$ but not in the vDG contributed to the cognitive-improving effect of EE-VEx in chronic inflammatory pain. $A$, The object-place recognition task. One object $\left(O_{B}\right)$ was moved to a novel place in the test phase, and the bias score was calculated as exploring times $\left(O_{B}-O_{A}\right) /\left(O_{B}+O_{A}\right)$ in sample and test phases. $B$, Impaired object-place recognition in mice with chronic inflammatory pain was reversed by EE-VEx. $n=8$ in each group. ${ }^{*} p<0.05$ vs saline group, ${ }^{\#} p<0.05$ vs CFA group, one-way ANOVA with Bonferroni's post hoc test. C, Ablating adult neurogenesis in the dDG but not in the vDG reversed EE-VEx's cognitive-enhancing effect. $n=8$ in each group. ${ }^{*} p<0.05, t$ test. $\boldsymbol{D}$, BDNF overexpression in the dDG improved object-place recognition of mice with chronic inflammatory pain. $n=8$ in each group. ${ }^{*} p<0.05, t$ test.

study (Fig. $1 B, C$ ). One intriguing finding is the limited effects of EE-VEx on baseline or acute nociceptive responses (Fig. $1 B, C$ ). This is consistent with one previous study on a similar inflammatory model in rats (Rossi and Neubert, 2008; Tall, 2009; Gabriel et al., 2010). However, using measurements other than reflex-based nociceptive responses yields some controversies (Rossi and Neubert, 2008; Tall, 2009; Gabriel et al., 2010), prompting us to evaluate other dimensions of pain as well.

We show anxiolytic (Fig. 1D-F) and cognitive-improving (Fig. 9B) effects of EE-VEx on chronic inflammatory mice. These findings indicate stronger effects of EE-VEx on central components of pain than peripheral responses. Consistently, we fail to observe significant local anti-inflammatory effects in CFAinjected paws by EE-VEx (Fig. 2). However, we do not exclude the possibility that EE-VEx alleviates widespread inflammatory responses triggered by CFA injection (Singhal et al., 2014), through which its analgesic, anxiolytic, and cognitive-enhancing effects are achieved.

Despite consistent beneficial effects of EE-VEx on pain by various reports (Vachon et al., 2013; Blázquez et al., 2014; Leger et al., 2015), the underlying mechanisms remain poorly understood. One well known neural consequence of EE-VEx is increased hippocampal neurogenesis (van Praag et al., 2000). We show that ablating adult neurogenesis in the VDG and dDG abolishes EE-VEx's beneficial effects on perceptual/affective (Figs. 5, 6) and cognitive (Fig. 9C) dimensions of pain, respectively. Thus, adult hippocampal neurogenesis at least partially contributes to EE-VEx's pain-relieving effects in chronic pain. The differential effects of neurogenesis ablation (with AAVDTA) or promotion (with BDNF) on pain and/or EE-VEx along the dorsoventral axis of the hippocampus parallel distinct gene expression, circuitry organization, electrophysiological properties, and behavioral functions of dorsal and ventral hippocampus (Bannerman et al., 2014). Similar functional dissociation of adult neurogenesis between $\mathrm{dDG}$ and vDG also has been reported (Sahay and Hen, 2007; O'Leary and Cryan, 2014). Increasing adult vDG neurogenesis alleviates depression and anxiety-like behaviors in rodents (Fox et al., 2006), both of which aggravate chronic pain symptoms (Bushnell et al., 2013; Craven et al., 2013). By contrast, neurogenesis in the dorsal pole is critical for declarative cognition (O’Leary and Cryan, 2014). 
We should note that mechanisms other than adult hippocampal neurogenesis cannot be excluded from EE-VEx's effects on chronic pain. EE-VEx also increases cortical gliogenesis (Ehninger and Kempermann, 2003), histone-tail acetylation (Fischer et al., 2007), and activities of several neurotransmitter systems including acetylcholine, dopamine, norepinephrine, and opioids (Sforzo et al., 1986; Fordyce and Farrar, 1991; Escorihuela et al., 1995; Soares et al., 1999; Lee et al., 2013). All of these factors could have influences on animal behaviors in chronic pain. In addition, combined environmental enrichment and exercise exert greater effects on both adult neurogenesis and animal behaviors than single paradigms alone (Fabel et al., 2009; Pham et al., 2010), indicating synergic effects of these components.

\section{Role of adult hippocampal neurogenesis in chronic pain}

Neurogenesis impairment has been observed under various chronic pain conditions (Duric and McCarson, 2006; Terada et al., 2008; Mutso et al., 2012; Dellarole et al., 2014; Dimitrov et al., 2014). In the present study, we show that chronic inflammatory pain decreases both the number of BrdU/DCX colabeled neurons and the dendrite complexity of DCX-labeled immature granular cells (Fig. 3). DCX is mainly expressed by migrating immature neurons in the adult brain (Gleeson et al., 1998; Gleeson et al., 1999) and is a reliable biomarker of newborn neurons in the subgranular cell zone of the DG (Brown et al., 2003).

Despite these consistent findings of impaired neurogenesis in chronic pain, controversies exist regarding their causal relationship (Apkarian et al., 2016; Kang et al., 2016; Zheng et al., 2016). In the present study, ablation of adult vDG neurogenesis aggravates thermal hyperalgesia in chronic inflammatory pain without affecting baseline or acute nociceptive responses (Fig. 5). In a recent study, however, ablating neurogenesis delayed the onset of tactile allodynia in mice with chronic neuropathic pain (Apkarian et al., 2016). Two important differences potentially underlie the discrepancy. First, inflammatory and neuropathic pain has a distinct central impact. Inflammatory pain is characterized by constant nociceptor activation, and anti-inflammatory responses initiate pain recovery (Kidd and Urban, 2001). By contrast, nerve injuries essentially cause a reorganization of the nervous system after loss of peripheral afferents and induce hypersensitive (central sensitization) pain memories (Costigan et al., 2009). Although accumulating evidence indicates distinct molecular mechanisms between inflammatory and neuropathic pain in the peripheral nervous system (Abrahamsen et al., 2008; Abdel et al., 2010), their central mechanistic differences are not fully understood. In general, neuropathic pain has a much stronger influence on the brain, such as more severe gray matter loss (Gustin et al., 2011), than non-neuropathic pain. Differential expression of hippocampal miRNAs has been reported in rat models of inflammatory and neuropathic pain (Hori et al., 2013). Potentially different contribution of hippocampal neurogenesis to these processes is open for investigation. Another significant difference between the present study and the study by Apkarian et al. (2016) is the method of pain measurement. Inflammatory and neuropathic pain involves both thermal hyperalgesia and mechanical allodynia. However, these signals are processed in distinct pathways. Whereas mechanical stimuli are preferentially transmitted by thick $A \beta$ fibers to the classical spinothalamic tract, thermal stimuli have a heavier impact on the thermo-regulatory system closely interconnected with the hippocampus (Craig, 2003). Consistently, we (Fig. 1 B,C) and others (Tall, 2009) observed stronger effects of EE-VEx on thermal hyperalgesia than mechanical allodynia in inflammatory pain. Overall, our current evidence suggests that the exact role of neurogenesis in pain modulation is multifaceted, depending on hippocampal subregions, pain models, and pain modalities.

\section{Clinical translation}

Although EE-VEx gains popularity in animal studies, this therapy is not easy to be standardized or controlled under clinical situations. Administration of exercise therapies on aged or severely painful patients is unrealistic and therefore poses limitations. However, many clinically applied analgesics and nondrug pain treatment such as environmental-behavioral therapies, acupuncture, noninvasive electrical stimulation, and dietary interventions impact hippocampal neurogenesis (Lledo et al., 2006; Kobelt et al., 2014; Nam et al., 2015; Heberden, 2016). Some antidepressants are recommended as first-line drugs for chronic pain management (Finnerup et al., 2015) and promote hippocampal neurogenesis (Malberg et al., 2000; Eisch and Petrik, 2012). Moreover, alleviation of chronic pain by antidepressants correlates with increased norepinephrine release in the hippocampus (Ignatowski and Spengler, 1994; Ignatowski et al., 2005), which benefits neurogenesis and prevents volume loss of hippocampus (Bremner et al., 2000). By contrast, long-term application of opiates inhibits hippocampal neurogenesis (Eisch et al., 2000) and frequently shows side effects, including tolerance and cognitive deficits (Christo, 2003). Interestingly, combinative application of reboxetine, a norepinephrine reuptake inhibitor, counteracts the antineurogenic and apoptotic effects of morphine (Meneghini et al., 2014). Thus, for preserving hippocampal neurogenesis in chronic pain treatments, combined pharmacological therapeutics with nonpharmacological paradigms such as EE-VEx could be of clinical significance. Indeed, there is already evidence that environmental enrichment and exercises increase norepinephrine and opioid signaling (Boecker et al., 2008; Lee et al., 2013) and enhance the analgesic effect of opioids (Smith et al., 2003, 2005).

In conclusion, the present study demonstrates distinct contribution of adult hippocampal neurogenesis along the dorsoventral axis to EE-VEx's beneficial effects on different dimensions of chronic pain and indicates combined pharmacological treatments with nonpharmacological paradigms, including EE-VEx as a promising option for further investigation on pain management.

\section{References}

Abdel Samad O, Liu Y, Yang FC, Kramer I, Arber S, Ma Q (2010) Characterization of two Runx1-dependent nociceptor differentiation programs necessary for inflammatory versus neuropathic pain. Mol Pain 6:45.

Abrahamsen B, Zhao J, Asante CO, Cendan CM, Marsh S, Martinez-Barbera JP, Nassar MA, Dickenson AH, Wood JN (2008) The cell and molecular basis of mechanical, cold, and inflammatory pain. Science 321:702-705.

Apkarian AV, Hashmi JA, Baliki MN (2011) Pain and the brain: specificity and plasticity of the brain in clinical chronic pain. Pain 152:S49-S64.

Apkarian AV, Mutso AA, Centeno MV, Kan L, Wu M, Levinstein M, Banisadr G, Gobeske KT, Miller RJ, Radulovic J, Hen R, Kessler JA (2016) Role of adult hippocampal neurogenesis in persistent pain. Pain 157:418-428.

Bannerman DM, Sprengel R, Sanderson DJ, McHugh SB, Rawlins JN, Monyer H, Seeburg PH (2014) Hippocampal synaptic plasticity, spatial memory and anxiety. Nat Rev Neurosci 15:181-192.

Bessa AL, Oliveira VN, Agostini GG, Oliveira RJS, Oliveira ACS, White GE, Wells GD, Teixeira DNS, Espindola FS (2016) Exercise intensity and recovery. J Strength Condition Res 30:311-319.

Blázquez G, Cañete T, Tobeña A, Giménez-Llort L, Fernández-Teruel A (2014) Cognitive and emotional profiles of aged Alzheimer's disease (3xTgAD) mice: effects of environmental enrichment and sexual dimorphism. Behav Brain Res 268:185-201.

Boecker H, Sprenger T, Spilker ME, Henriksen G, Koppenhoefer M, Wagner 
KJ, Valet M, Berthele A, Tolle TR (2008) The runner's high: opioidergic mechanisms in the human brain. Cereb Cortex 18:2523-2531.

Bremner JD, Narayan M, Anderson ER, Staib LH, Miller HL, Charney DS (2000) Hippocampal volume reduction in major depression. Am J Psychiatry 157:115-118.

Brown JP, Couillard-Després S, Cooper-Kuhn CM, Winkler J, Aigner L, Kuhn HG (2003) Transient expression of doublecortin during adult neurogenesis. J Comp Neurol 467:1-10.

Bushnell MC, Ceko M, Low LA (2013a) Cognitive and emotional control of pain and its disruption in chronic pain. Nat Rev Neurosci 14:502-511.

Chaplan SR, Bach FW, Pogrel JW, Chung JM, Yaksh TL (1994) Quantitative assessment of tactile allodynia in the rat paw. J Neurosci Methods 53:55-63.

Christo PJ (2003) Opioid effectiveness and side effects in chronic pain. Anesthesiol Clin North Am 21:699-713.

Costigan M, Scholz J, Woolf CJ (2009) Neuropathic pain: a maladaptive response of the nervous system to damage. Annu Rev Neurosci 32:1-32.

Craig AD (2003) Interoception: the sense of the physiological condition of the body. Curr Opin Neurobiol 13:500-505.

Craven P, Cinar O, Madsen T (2013) Patient anxiety may influence the efficacy of ED pain management. Am J Emerg Med 31:313-318.

Dellarole A, Morton P, Brambilla R, Walters W, Summers S, Bernardes D, Grilli M, Bethea JR (2014) Neuropathic pain-induced depressive-like behavior and hippocampal neurogenesis and plasticity are dependent on TNFR1 signaling. Brain Behav Immun 41:65-81.

Dimitrov EL, Tsuda MC, Cameron HA, Usdin TB (2014) Anxiety- and depression-like behavior and impaired neurogenesis evoked by peripheral neuropathy persist following resolution of prolonged tactile hypersensitivity. J Neurosci 34:12304-12312.

Duric V, McCarson KE (2006) Persistent pain produces stress-like alterations in hippocampal neurogenesis and gene expression. J Pain 7:544-555.

Ehninger D, Kempermann G (2003) Regional effects of wheel running and environmental enrichment on cell genesis and microglia proliferation in the adult murine neocortex. Cereb Cortex 13:845-851.

Eisch AJ, Petrik D (2012) Depression and hippocampal neurogenesis: a road to remission? Science 338:72-75.

Eisch AJ, Barrot M, Schad CA, Self DW, Nestler EJ (2000) Opiates inhibit neurogenesis in the adult rat hippocampus. Proc Natl Acad Sci U S A 97:7579-7584.

Escorihuela RM, Fernández-Teruel A, Tobeña A, Vivas NM, Mármol F, Badia A, Dierssen M (1995) Early environmental stimulation produces longlasting changes on beta-adrenoceptor transduction system. Neurobiol Learn Mem 64:49-57.

Fabel K, Wolf SA, Ehninger D, Babu H, Leal-Galicia P, Kempermann G (2009) Additive effects of physical exercise and environmental enrichment on adult hippocampal neurogenesis in mice. Front Neurosci 3:50.

Fan D, Li J, Zheng B, Hua L, Zuo Z (2016) Enriched environment attenuates surgery-induced impairment of learning, memory, and neurogenesis possibly by preserving BDNF expression. Mol Neurobiol 53:344-354.

Finnerup NB, Attal N, Haroutounian S, McNicol E, Baron R, Dworkin RH, Gilron I, Haanpää M, Hansson P, Jensen TS, Kamerman PR, Lund K, Moore A, Raja SN, Rice AS, Rowbotham M, Sena E, Siddall P, Smith $\mathrm{BH}$, Wallace M (2015) Pharmacotherapy for neuropathic pain in adults: a systematic review and meta-analysis. Lancet Neurol 14:162-173.

Fischer A, Sananbenesi F, Wang X, Dobbin M, Tsai LH (2007) Recovery of learning and memory is associated with chromatin remodeling. Nature 447:178-182.

Fordyce DE, Farrar RP (1991) Physical activity effects on hippocampal and parietal cortical cholinergic function and spatial learning in F344 rats. Behav Brain Res 43:115-123.

Fox C, Merali Z, Harrison C (2006) Therapeutic and protective effect of environmental enrichment against psychogenic and neurogenic stress. Behav Brain Res 175:1-8.

Gabriel AF, Paoletti G, Della Seta D, Panelli R, Marcus MA, Farabollini F, Carli G, Joosten EA (2010) Enriched environment and the recovery from inflammatory pain: social versus physical aspects and their interaction. Behav Brain Res 208:90-95.

Gleeson JG, Allen KM, Fox JW, Lamperti ED, Berkovic S, Scheffer I, Cooper EC, Dobyns WB, Minnerath SR, Ross ME, Walsh CA (1998) Doublecortin, a brain-specific gene mutated in human X-linked lissencephaly and double cortex syndrome, encodes a putative signaling protein. Cell 92:63-72.

Gleeson JG, Lin PT, Flanagan LA, Walsh CA (1999) Doublecortin is a microtubule-associated protein and is expressed widely by migrating neurons. Neuron 23:257-271.

Gustin SM, Peck CC, Wilcox SL, Nash PG, Murray GM, Henderson LA (2011) Different pain, different brain: thalamic anatomy in neuropathic and non-neuropathic chronic pain syndromes. J Neurosci 31:5956-5964.

Heberden C (2016) Modulating adult neurogenesis through dietary interventions. Nutr Res Rev 29:163-171.

Hill AS, Sahay A, Hen R (2015) Increasing adult hippocampal neurogenesis is sufficient to reduce anxiety and depression-like behaviors. Neuropsychopharmacology 40:2368-2378.

Hori Y, Goto G, Arai-Iwasaki M, Ishikawa M, Sakamoto A (2013) Differential expression of rat hippocampal microRNAs in two rat models of chronic pain. Int J Mol Med 32:1287-1292.

Ignatowski TA, Spengler RN (1994) Tumor necrosis factor-alpha: presynaptic sensitivity is modified after antidepressant drug administration. Brain Res 665:293-299.

Ignatowski TA, Sud R, Reynolds JL, Knight PR, Spengler RN (2005) The dissipation of neuropathic pain paradoxically involves the presence of tumor necrosis factor-alpha (TNF). Neuropharmacology 48:448-460.

Kang E, Wen Z, Song H, Christian KM, Ming GL (2016) Adult neurogenesis and psychiatric disorders. Cold Spring Harb Perspect Biol 8:a019026.

Kidd BL, Urban LA (2001) Mechanisms of inflammatory pain. Br J Anaesth 87:3-11.

Kobelt LJ, Wilkinson AE, McCormick AM, Willits RK, Leipzig ND (2014) Short duration electrical stimulation to enhance neurite outgrowth and maturation of adult neural stem progenitor cells. Ann Biomed Eng 42:2164-2176.

Lee MY, Yu JH, Kim JY, Seo JH, Park ES, Kim CH, Kim H, Cho SR (2013) Alteration of synaptic activity-regulating genes underlying functional improvement by long-term exposure to an enriched environment in the adult brain. Neurorehabil Neural Repair 27:561-574.

Leger M, Paizanis E, Dzahini K, Quiedeville A, Bouet V, Cassel JC, Freret T, Schumann-Bard P, Boulouard M (2015) Environmental enrichment duration differentially affects behavior and neuroplasticity in adult mice. Cereb Cortex 25:4048-4061.

Liu MG, Chen J (2009) Roles of the hippocampal formation in pain information processing. Neurosci Bull 25:237-266.

Lledo PM, Alonso M, Grubb MS (2006) Adult neurogenesis and functional plasticity in neuronal circuits. Nat Rev Neurosci 7:179-193.

Malberg JE, Eisch AJ, Nestler EJ, Duman RS (2000) Chronic antidepressant treatment increases neurogenesis in adult rat hippocampus. J Neurosci 20:9104-9110.

Meneghini V, Cuccurazzu B, Bortolotto V, Ramazzotti V, Ubezio F, Tzschentke TM, Canonico PL, Grilli M (2014) The noradrenergic component in tapentadol action counteracts mu-opioid receptor-mediated adverse effects on adult neurogenesis. Mol Pharmacol 85:658-670.

Mutso AA, Radzicki D, Baliki MN, Huang L, Banisadr G, Centeno MV, Radulovic J, Martina M, Miller RJ, Apkarian AV (2012) Abnormalities in hippocampal functioning with persistent pain. J Neurosci 32:5747-5756.

Nakashiba T, Cushman JD, Pelkey KA, Renaudineau S, Buhl DL, McHugh TJ, Rodriguez Barrera V, Chittajallu R, Iwamoto KS, McBain CJ, Fanselow MS, Tonegawa S (2012) Young dentate granule cells mediate pattern separation, whereas old granule cells facilitate pattern completion. Cell 149:188-201.

Nam MH, Ahn KS, Choi SH (2015) Acupuncture: a potent therapeutic tool for inducing adult neurogenesis. Neural Regen Res 10:33-35.

Nithianantharajah J, Hannan AJ (2006) Enriched environments, experience-dependent plasticity and disorders of the nervous system. Nat Rev Neurosci 7:697-709.

O'Leary OF, Cryan JF (2014) A ventral view on antidepressant action: roles for adult hippocampal neurogenesis along the dorsoventral axis. Trends Pharmacol Sci 35:675-687.

Paxinos G, Franklin KBJ (2001) The mouse brain in stereotaxic coordinates. San Diego: Academic.

Pham TM, Hagman B, Codita A, Van Loo PL, Strommer L, Baumans V (2010) Housing environment influences the need for pain relief during post-operative recovery in mice. Physiol Behav 99:663-668.

Rosenzweig S, Wojtowicz JM (2011) Analyzing dendritic growth in a pop- 
ulation of immature neurons in the adult dentate gyrus using laminar quantification of disjointed dendrites. Front Neurosci 5:34.

Rossi HL, Neubert JK (2008) Effects of environmental enrichment on thermal sensitivity in an operant orofacial pain assay. Behav Brain Res 187:478-482.

Sahay A, Hen R (2007) Adult hippocampal neurogenesis in depression. Nat Neurosci 10:1110-1115.

Sforzo GA, Seeger TF, Pert CB, Pert A, Dotson CO (1986) In vivo opioid receptor occupation in the rat brain following exercise. Med Sci Sports Exerc 18:380-384.

Singhal G, Jaehne EJ, Corrigan F, Baune BT (2014) Cellular and molecular mechanisms of immunomodulation in the brain through environmental enrichment. Front Cell Neurosci 8:97.

Smith MA, Bryant PA, McClean JM (2003) Social and environmental enrichment enhances sensitivity to the effects of kappa opioids: studies on antinociception, diuresis and conditioned place preference. Pharmacol Biochem Behav 76:93-101.

Smith MA, Chisholm KA, Bryant PA, Greene JL, McClean JM, Stoops WW, Yancey DL (2005) Social and environmental influences on opioid sensitivity in rats: importance of an opioid's relative efficacy at the mu-receptor. Psychopharmacology (Berl) 181:27-37.

Soares J, Holmes PV, Renner KJ, Edwards GL, Bunnell BN, Dishman RK (1999) Brain noradrenergic responses to footshock after chronic activitywheel running. Behav Neurosci 113:558-566.

Soumier A, Sibille E (2014) Opposing effects of acute versus chronic blockade of frontal cortex somatostatin-positive inhibitory neurons on behavioral emotionality in mice. Neuropsychopharmacology 39:2252-2262.

Tall JM (2009) Housing supplementation decreases the magnitude of inflammation-induced nociception in rats. Behav Brain Res 197:230-233.
Terada M, Kuzumaki N, Hareyama N, Imai S, Niikura K, Narita M, Yamazaki M, Suzuki T, Narita M (2008) Suppression of enriched environmentinduced neurogenesis in a rodent model of neuropathic pain. Neurosci Lett 440:314-318.

Vachon P, Millecamps M, Low L, Thompson SJ, Pailleux F, Beaudry F, Bushnell CM, Stone LS (2013) Alleviation of chronic neuropathic pain by environmental enrichment in mice well after the establishment of chronic pain. Behav Brain Funct 9:22.

van Praag H, Kempermann G, Gage FH (1999) Running increases cell proliferation and neurogenesis in the adult mouse dentate gyrus. Nat Neurosci 2:266-270.

van Praag H, Kempermann G, Gage FH (2000) Neural consequences of environmental enrichment. Nat Rev Neurosci 1:191-198.

Vedovelli K, Silveira E, Velho E, Stertz L, Kapczinski F, Schröder N, Bromberg E (2011) Effects of increased opportunity for physical exercise and learning experiences on recognition memory and brain-derived neurotrophic factor levels in brain and serum of rats. Neuroscience 199:284-291.

Whissell PD, Rosenzweig S, Lecker I, Wang DS, Wojtowicz JM, Orser BA (2013) Gamma-aminobutyric acid type A receptors that contain the delta subunit promote memory and neurogenesis in the dentate gyrus. Ann Neurol 74:611-621.

Williams AC, Craig KD (2016) Updating the definition of pain. Pain 157:2420-2423.

Wu MV, Hen R (2014) Functional dissociation of adult-born neurons along the dorsoventral axis of the dentate gyrus. Hippocampus 24:751-761.

Zhang Y, Liu FY, Liao FF, Wan Y, Yi M (2014) Exacerbation of tonic but not phasic pain by entorhinal cortex lesions. Neurosci Lett 581:137-142.

Zheng J, Yi M, Wan Y (2016) Hippocampal neurogenesis: does it relieve or worsen chronic pain? Pain 157:506-507. 